\title{
Acoustic mapping of ocean currents using networked distributed sensors
}

\author{
Chen-Fen Huang \\ Institute of Oceanography, National Taiwan University, Taipei 10617, Taiwan \\ T. C. Yang ${ }^{\text {a) }}$ and Jin-Yuan Liu ${ }^{\text {b) }}$ \\ Institute of Applied Marine Physics and Undersea Technology, National Sun Yat-sen University, \\ Kaohsiung 80424, Taiwan \\ Jeff Schindall \\ Naval Research Laboratory, 4555 Overlook Avenue SW, Washington, DC 20375
}

(Received 23 November 2012; revised 8 July 2013; accepted 18 July 2013)

\begin{abstract}
Distributed underwater sensors are expected to provide oceanographic monitoring over large areas. As fabrication technology advances, low cost sensors will be available for many uses. The sensors communicate to each other and are networked using acoustic communications. This paper first studies the performance of such systems for current measurements using tomographic inversion approaches to compare with that of a conventional system which distributes the sensors on the periphery of the area of interest. It then proposes two simple signal processing methods for ocean current mapping (using distributed networked sensors) aimed at real-time in-buoy processing. Tomographic inversion generally requires solving a challenging high dimensional inverse problem, involving substantial computations. Given distributed sensors, currents can be constructed locally based on data from neighboring sensors. It is shown using simulated data that similar results are obtained using distributed processing as using conventional tomographic approaches. The advantage for distributed systems is that by increasing the number of nodes, one gains a much more improved performance. Furthermore, distributed systems use much less energy than a conventional tomographic system for the same area coverage. Experimental data from an acoustic communication and networking experiment are used to demonstrate the feasibility of acoustic current mapping. (C) 2013 Acoustical Society of America. [http://dx.doi.org/10.1121/1.4817835]
\end{abstract}

PACS number(s): 43.60.Rw, 43.60.Pt [ZHM]

Pages: 2090-2105

\section{INTRODUCTION}

Acoustical oceanography uses acoustic measurements to invert for ocean environmental changes, thus allowing monitoring of oceanographic features (eddies, currents, etc.), detection/sensing of underwater seismic events (earthquakes and tidal waves), and so on. One such method is Ocean Acoustic Tomography (OAT), proposed by Munk and Wunsch in 1979, which uses (the perturbation of) acoustic ray travel time to invert and map mesoscale oceanic disturbance in deep ocean. ${ }^{1,2}$ To reach long distances (hundreds of kilometers), low frequency (tens to hundreds of hertz) sources and receivers were used; the equipment was heavy and the measurements were costly to conduct, often requiring ship support, especially for moving ship tomography ${ }^{3}$ ). Coastal acoustic tomography (CAT) was recently proposed as the application of OAT to coastal seas, with the goal of continuously monitoring the tidal currents in harbors, bays, straits, and inland seas, ${ }^{4-7}$ based on travel time differences between reciprocal transmissions. The sources and receivers are deployed in remote areas without risk or inconvenience

\footnotetext{
a) Author to whom correspondence should be addressed. Electronic mail: tsihyang@gmail.com

b) Also at: National Taitung University, Taitung 95092, Taiwan.
}

to shipping, fisheries, or marine aquaculture industries. Because of the shorter (tens of kilometers) distances, higher (kilohertz) frequency sources and receivers can be employed. The sensors are usually moored to the ocean bottom and the data are cabled back to shore.

Future underwater sensing is envisioned using inexpensive (thus expendable) underwater sensors distributed over a wide area, deployed close to the bottom or at a sufficient depth, and networked together using underwater acoustic communications. Sensor data can be relayed to the base station via the acoustic network. (Acoustic sensing and networking may use different, non-overlapping frequency bands based on the need.) As fabrication technology advances, many low cost sensors will be available. This system concept, referred to as the Distributed Networked Underwater Sensors (DNUS) system in this paper, has attracted a great deal of attention recently from the point of view of target detection, since the probability of detection increases with the number of sensors, denoted as $N$. In other words, as $N$ increases, there is a high probability that some sensors will be close to the events and will return with high quality (high signal-to-noise ratio) data. Likewise, with a larger number of sensors one can collect oceanographic/seismic, biological, and chemical data over a wide area for weather prediction, disaster prevention, pollution 


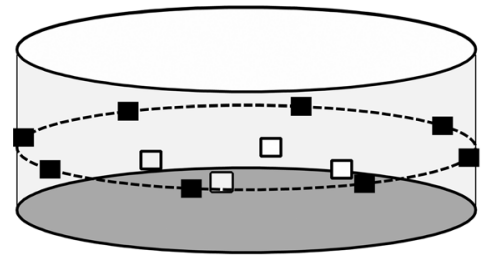

(b)

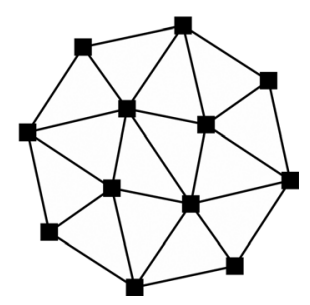

FIG. 1. (a) 3D and (b) top view of a distributed sensor network.

monitoring, offshore oil exploration, and so on. (At the present time such data are available only for limited areas.) The key considerations for distributed sensing are easy deployment (using a light weight sensor) and low power consumption as each sensor carries its own power supply. Signal processing for such systems is likely to require new approaches (preferably in-buoy processing to reduce the communication load) that are significantly different from the traditional approaches based on beamforming which assumes that the signals over (co-located) receivers are spatially coherent. This paper addresses the problem of how to use networked distributed sensors for wide area current monitoring. The distributed current mapping system concept and signal processing methodology are developed in this paper. The performance of the system is studied using simulated current field data to determine the mapping accuracy. The potential of such a system to map the current over a wide area and continuously as a function of time is demonstrated with real ocean data. The inverted current field as a function of time is found in reasonable agreement with that estimated from a co-located Acoustic Doppler Current Profiler (ADCP).

The distributed current sensing system concept is discussed in Sec. II, illustrated by candidate sensor configurations. The total power for sound projection is analyzed in Sec. III as a function of the number of sensors and acoustic frequency used. A conventional tomographic inversion algorithm is applied in Sec. IV to selected distributed systems to compare their performance with that of the conventional OAT system (in terms of errors between the estimated and true current fields). It provides some clues as to how many distributed sensors are needed to achieve the same performance as that of a conventional OAT system. In Sec. V, simple signal processing methods (algorithms) are proposed for distributed systems aimed at real-time in-buoy processing of data over a wide area (without requiring a powerful computer as is the case for OAT). The algorithms are shown to yield a comparable performance as that obtained with the tomographic inversion methods (Sec. IV) under certain conditions. At sea data are analyzed in Sec. VI using distributed signal processing to compare with the ground truth, demonstrating the feasibility of continuous mapping of ocean currents over an area. A short summary is given in Sec. VII.

\section{DISTRIBUTED SENSING OF CURRENT FIELD: DESIGN CONSIDERATIONS}

Following the tomographic concept, traditional OAT/ CAT systems deploy the sensors (functioning both as (a) $\mathrm{N}=8$

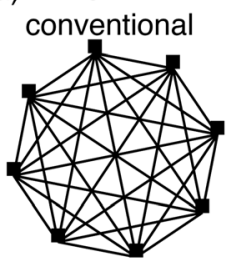

(d) $\mathrm{N}=30, \mathrm{M}=43$, $\rho=0.245$

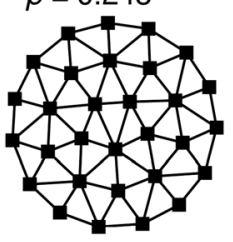

(b) $\mathrm{N}=12, \mathrm{M}=14$, $\rho=0.43$

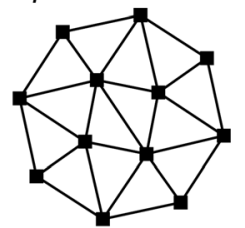

(e) $\mathrm{N}=42, \mathrm{M}=64$, $\rho=0.216$

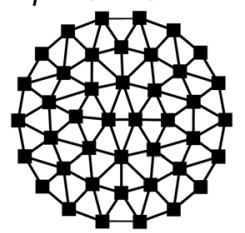

(c) $\mathrm{N}=16, \mathrm{M}=20$, $\rho=0.363$

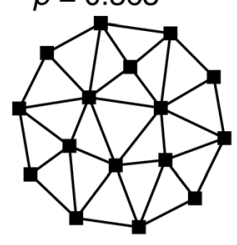

(f) $\mathrm{N}=49, \mathrm{M}=76$, $\rho=0.187$

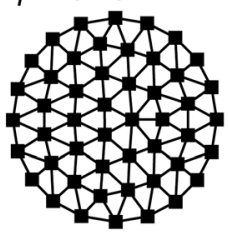

FIG. 2. Examples of sensor configurations for (a) the conventional OAT system and (b)-(f) DNUS systems. The lines indicate the communication/propagation routes. $N=$ total number of nodes, $M=$ total number of triangles for a given DNUS, and $\rho=r_{0} / D=$ the maximum node separation divided by the diameter.

sources and receivers) on the boundary (periphery) looking into the volume to sense the activities inside. For example, the sensors can be deployed on a circle as shown by the (eight) solid squares in Fig. 1(a). Given (four) more sensors, one has a choice to deploy the additional sensors on the circle or deploy them within the circle as shown by the open squares in Fig. 1(a). Viewed in three dimensions, the sensors are deployed close to the ocean bottom to perform tomographic measurements of activities in the ocean volume above. Estimation of the three-dimensional (3D) current distribution requires full field tomographic inversion. Viewed in two dimensions [the top view in Fig. 1(b)], the sensors deployed inside the circle together with those on the circle form a distributed system, since the sensors are approximately uniformly distributed (per area). Examples of distributed sensors are shown in Figs. 2(b)-2(f). For comparison, a traditional tomographic system with eight sensors is shown in Fig. 2(a).

For underwater distributed sensing, the information collected will be relayed to other nodes using acoustic communications. The distributed sensors form an acoustic communication network to pass on node identification and position information, command and control messages as well as (reduced) acoustic sensor data. ${ }^{8}$ Each node in Figs. 2(b)-2(f) in principle talks only to the neighboring nodes to minimize interference to communications between other pairs of nodes. (When one or more nodes fail, the neighbor nodes will then increase power to communicate to the surviving nodes at a longer distance.) In contrast, the sensors in the traditional OAT [Fig. 2(a)] must have sufficient power to transmit its signal to all sensors on the circle. The solid lines in Fig. 2 indicate the communication (signal propagation) routes between the nodes (sensors). For an individual sensor, it takes much more power to transmit a signal in the traditional OAT [Fig. 2(a)] system than in a DNUS system [Figs. 2(b)-2(f)] due to the much longer distances the signal needs to travel while maintaining the same signal quality at the receiver, ${ }^{9}$ i.e., the received signal-to-noise ratio (SNR). For the 
entire system, the total signal transmission power is dependent on the frequency used, node separation, and the total number of nodes as analyzed in Sec. III.

It is apparent from Fig. 2 that the DNUS consists of many triangles connected by the neighboring nodes (via underwater acoustic communications). In other words, the triangle can be considered the basic building block for a DNUS system. Accordingly, the signal processing for DNUS will be based on the triangle as discussed below in Sec. V. Since a triangle with a small angle yields a high error in current measurements (Sec. V A), to optimize the measurements the design goal is to maximize all angles of all triangles for each DNUS. [The optimum topology is the equilateral triangle to balance the power projection in all three directions and allow the current field to be viewed equally in all angles using the projection slice theorem. However, one cannot construct a (two-dimensional) circle based on equilateral triangles and in practice the nodes are not likely to be deployed in equilateral triangles either.] Given a circular area of diameter $D$ and an approximate transmission distance $r_{0}$ between the nodes, the node locations can be found by solving for equilibrium in a truss structure (by piecewise linear force-displacement relations) and reset the topology by the Delaunay triangulation. ${ }^{10}$ Figures 2(b)-2(f) are obtained using this algorithm.

Using DNUS for current measurements, the system performance depends on the following parameters: The diameter of the area to be surveyed, the node separation, the acoustic (carrier) frequency and bandwidth (BW) to be used, the total power and the spatial resolution of the system, etc. In general, the BW determines the temporal resolution and consequently the accuracy of the current measurements. The optimum carrier frequency will be determined by the node separation for the purpose of minimizing the transmission loss while maintaining a minimum SNR at the receiver as analyzed in Sec. III B below. Typically, the smaller the node separation, the higher the carrier frequency. For DNUS, it is obvious from Figs. 2(b)-2(f), that the smaller the node separation, the higher the spatial resolution. To determine the spatial resolution for the conventional OAT system, an extensive inversion analysis will be required using the resolution map. ${ }^{11}$

For long term ocean monitoring (of a given area), power consumption is an important issue, particularly when operated on battery power. For the conventional OAT system, the total power increases with the number of sensors used. For the DNUS system, one finds: (1) While more nodes will be needed when the node separation decreases, the total power also decreases with an increasing number of sensors (see Sec. III). As a result, distributed sensor systems use much less transmitting power than a corresponding OAT system (Sec. III). (2) By increasing the number of sensors, distributed systems can achieve a similar or better performance compared with the OAT system (Sec. IV). While conventional OAT methods can (also) be applied to distributed sensor systems, they generally require intensive computations and hence a much higher computational power (usually carried out at a centralized unit). Distributed signal processing, given in Sec. V, is simple and can be conducted at each node using in-buoy processing at a small loss of performance. From the power point of view, DNUS systems achieve a much longer lifetime for a battery-powered system compared with the traditional OAT system by saving in power for signal projection.

The DNUS system offers several features which are desirable from the system point of view. (1) For the DNUS system, if one node fails, it affects only the local measurement with negligible/no impact on measurements in other areas. In this case, neighboring functional nodes will be connected forming larger triangles, leaving other triangles unaffected. This feature becomes attractive when many sensor nodes are deployed, each providing backup or redundant data for the other. (2) For DNUS, sensor locations are continuously monitored/estimated using high frequency communication (probe) signals. (Range information is already provided in many commercial modems.) A traditional OAT system often uses low frequency signals to reach distant nodes. Additional high frequency transponders are then required to measure the sensor locations. (3) DNUS sensor topology can be adjusted adaptively according to the (expected) current distribution. For example, more nodes can be deployed in areas where the spatial variations of the currents are expected to be high. The traditional OAT is less adaptive. The sensors are distributed on the periphery; only their spacing can be adjusted. It requires many inversion analyses to determine the optimal spacing.

From the system point of view, since the spatial resolution increases with the number of nodes deployed and the total power decreases with an increasing number of nodes, it is envisioned that future DNUS will involve many sensor nodes [e.g., Figs. 2(e) and 2(f)], connected by a high frequency acoustic communication network (here we ignore the sensor cost assuming it is not an issue). From the science point of view, one needs to establish some yardsticks regarding the performance of the traditional OAT system and the distributed systems under different current distributions. For this purpose, we shall evaluate the performance of the OAT and distributed systems given in Figs. 2(a) and 2(b) using simulated data (in Sec. IV) to illustrate the underlying physics.

\section{SOUND PROJECTION}

In this section, we estimate and compare the total power required to transmit a broadband signal for a conventional OAT [Fig. 2(a)] and the distributed systems depicted in Figs. 2(b)-2(f). Next, we determine the optimal frequency to be used for a DNUS system from the point of view of minimizing the total transmission power.

\section{A. Transmission power analysis}

The basic criterion for the power analysis is that the signal, whichever node it comes from, should be received at whatever receiver with a minimum SNR equal to or greater than, say, $\mathrm{SNR}_{0}=20 \mathrm{~dB}$ (for a conservative estimate to allow some performance degradation in the real world). For an individual sensor (acting as a source), the minimum power required to transmit a broadband signal with a $\mathrm{BW}$ is given in $\mathrm{dB}$ by 


$$
\begin{aligned}
P\left(r_{0} ; f\right)= & \mathrm{SNR}_{0}+\mathrm{TL}\left(r_{0} ; f\right)+\mathrm{NL}(f) \\
& +10 \log \mathrm{BW}-\mathrm{PG} .
\end{aligned}
$$

The various terms in the above equation are defined as follows: $\mathrm{PG}$ is the processing gain, $\mathrm{PG}=10 \log (\mathrm{T} \cdot \mathrm{BW})$, where $\mathrm{T}$ is the duration, and $\mathrm{BW}$ is the bandwidth of the signal; $\mathrm{NL}(f)$ is the ambient noise level per Hertz at the center frequency $f ; \operatorname{TL}\left(r_{0} ; f\right)$ is the transmission loss for a signal to travel range $r_{0}$. To simplify the calculation, we have ignored the frequency dependence of the TL and NL within the signal band. The range $r_{0}$ should be determined by the maximum range of all ranges between adjacent nodes. As shown in Fig. 2, one has $r_{0}=0.43 D, 0.36 D, 0.25 D, 0.22 D$, and $0.19 D$ for Figs. 2(b)-2(f), respectively, where $D$ is the diameter of the circle. The total power required to transmit a signal from all nodes in a DNUS system is then $P_{N}\left(r_{0} ; f\right)$ $=P\left(r_{0} ; f\right)+10 \log N$, where $N$ is the total number of nodes in the DNUS. In comparison, the total power needed for signal transmission for an OAT system is given by $P_{N}(D ; f)=P(D ; f)+10 \log N$.

For numerical illustrations of the minimum required source power, we assume that the sensors transmit a broadband binary phase shift keying signal centered at $4 \mathrm{kHz}$ with a bandwidth of $4 \mathrm{kHz}$, consisting of 511 bits of $m$-sequence. The signal yields a $P G=27 \mathrm{~dB}$. The $\mathrm{TL}$ is in general dependent on the acoustic environment (water depth, sound speed, and sediment profiles), and needs to be determined using a propagation model. For shallow water propagation, the TL usually obeys spherical spreading to a range approximately equal to the water depth followed by cylindrical spreading beyond that point, plus attenuation due to water absorption and bottom scattering. For our purposes, in order to carry out analytic calculations, we shall assume a simplified expression for the depth averaged TL given by $\mathrm{TL}(r ; f)=k \log (r)+0.001 \alpha r$, where $r$ is measured in meter, $k$ is the spreading loss factor, and $\alpha$ is the attenuation coefficient of sea water in $\mathrm{dB} / \mathrm{km}$. Using a propagation model, one finds that the depth averaged TL for a typical shallow water environment can be fitted with $k \sim 17$ (at $4 \mathrm{kHz}$ ). This value of $k$ will be assumed for other frequencies as well; other values of $k$ can be substituted if needed. For the attenuation, a formula recently proposed by Ainslie and $\mathrm{McColm}^{12}$ will be used here. The model takes into account not only the effect of temperature and depth on the absorption coefficient but also the relaxation frequencies caused by boric acid and magnesium sulfate. In our calculations, we assumed a salinity of $35 \mathrm{ppm}$, a water temperature of $20^{\circ} \mathrm{C}$, an acidity of 8 , and a water depth of $100 \mathrm{~m}$ for a typical shallow water environment. One finds that $\alpha$ is $0.242 \mathrm{~dB} / \mathrm{km}$ at $4 \mathrm{kHz}$. For the ambient noise within 1 to $50 \mathrm{kHz}$, the noise is predominantly wind-driven. Based on the Wenz curve, one has $\mathrm{NL}(w, f)=50+7.5 w^{1 / 2}+20 \log f-40 \log (f+0.4)$ in $\mathrm{dB} / \mathrm{Hz}$. Using a wind speed $w=5 \mathrm{~m} / \mathrm{s}$, for example, one finds $\mathrm{NL}=53 \mathrm{~dB} / \mathrm{Hz}$ at $4 \mathrm{kHz}$.

Given these values, one plots the total power of an OAT system in Fig. 3 for three cases of $D: D=10,15$, and $20 \mathrm{~km}$. As expected, the total power of the OAT system increases logarithmically with the number of sensors deployed as shown by the left set of curves (solid symbols) in Fig. 3(a) for $N=5,8,10$, and 12. On the other hand, one finds that the total power of the DNUS system decreases slightly with the number of sensors deployed, as shown by the right sets of curves (open symbols) in Fig. 3(a) for $N=12,16,30,42$, and 49 for the DNUS shown in Figs. 2(b)-2(f), respectively. This result [Fig. 3(a)] shows that the DNUS uses significantly less total power than the OAT system projecting at $4 \mathrm{kHz}$, which is not a surprise. More power savings can be achieved for the DNUS system by using higher frequencies as discussed in Sec. III B.

\section{B. Optimal projecting frequency}

Next we discuss the optimal (carrier) frequency for the DNUS system from the point of view of minimizing the total projection power. What frequency to use is dependent on $r_{0}$, the largest separation between nodes in a given sensor configuration. The optimal frequency for a DNUS system can be determined by balancing the frequency dependence of $\operatorname{TL}\left(r_{0} ; f\right)$ against that of $\mathrm{NL}(f)$ using the following equation: $P_{N}\left(r_{0} ; f\right)-P_{N}\left(r_{0}\right.$; $\left.f_{0}\right)=P\left(r_{0} ; f\right)-P\left(r_{0} ; f_{0}\right)$, where
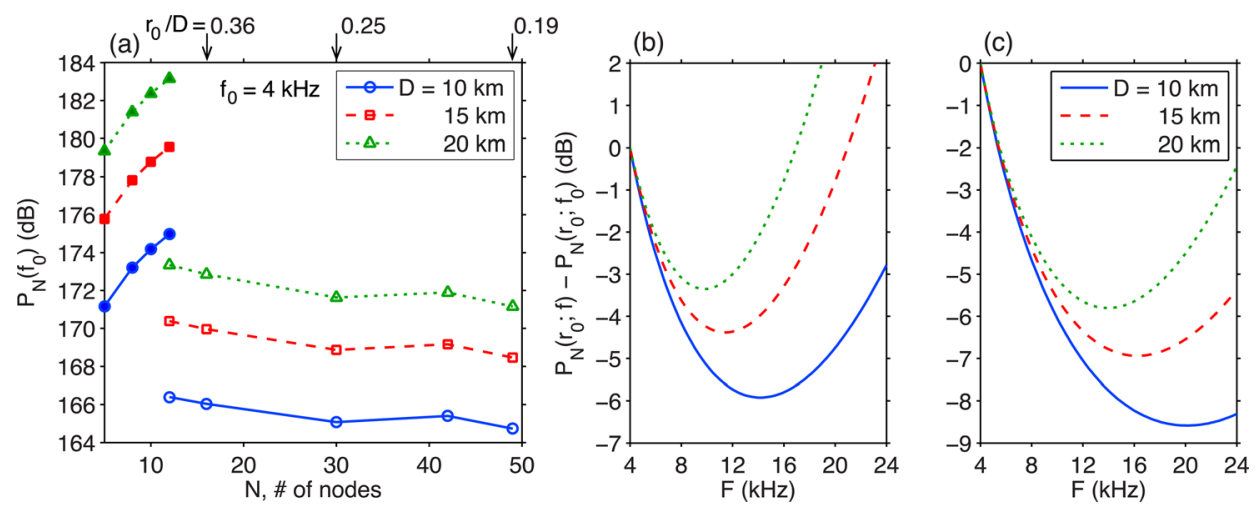

FIG. 3. (Color online) (a) Total transmission power versus the number of nodes $N$ to achieve a minimum $20 \mathrm{~dB}$ of SNR at each receiver. The left set of curves (solid symbols) are for the sensors deployed on the periphery of the circle (the conventional OAT system), $N=5,8,10$, and 12 . The right set of curves (open symbols) are for the DNUS systems with different $N$ as shown in Figs. 2(b)-2(f). (b) The frequency dependence of the total transmission power for the sensor configuration given in Fig. 2(c). (c) The frequency dependence of the total transmission power for the sensor configuration given in Fig. 2(f). 


$$
\begin{aligned}
P\left(r_{0} ; f\right)-P\left(r_{0} ; f_{0}\right)= & \operatorname{TL}\left(r_{0} ; f\right)-\operatorname{TL}\left(r_{0} ; f_{0}\right) \\
& +\operatorname{NL}(f)-\operatorname{NL}\left(f_{0}\right) \\
= & 0.001\left[\alpha(f)-\alpha\left(f_{0}\right)\right] r_{0} \\
& +\operatorname{NL}(f)-\operatorname{NL}\left(f_{0}\right) .
\end{aligned}
$$

Given that the total power of the DNUS system at $4 \mathrm{kHz}$ is approximately constant for all $N$, one can use this as a reference, i.e., $f_{0}=4 \mathrm{kHz}$, to project the total power at other frequencies. Consider the sensor configuration as given in Fig. 2(c), for example, where $r_{0}=3.63,5.45$, and $7.26 \mathrm{~km}$ for $D=10,15$, and $20 \mathrm{~km}$, respectively. For each $D$, one calculates $P(f)-P\left(f_{0}\right)$ and displays it as a function of frequency $f$, where $f_{0}=4 \mathrm{kHz}$, as shown in Fig. 3(b). One finds for $D=10 \mathrm{~km}$, the optimum frequency for this configuration is $f$ $\sim 14 \mathrm{kHz}$. One gains a saving of $\sim 6 \mathrm{~dB}$ using this frequency instead of $4 \mathrm{kHz}$. For $D=15 \mathrm{~km}$, the optimum frequency for this configuration is $f \sim 12 \mathrm{kHz}$. One gains a saving of $\sim 4.4 \mathrm{~dB}$ using this frequency instead of $4 \mathrm{kHz}$. For $D=20 \mathrm{~km}$, the optimum frequency for this configuration is $f \sim 10 \mathrm{kHz}$. One gains a saving of $\sim 3.4 \mathrm{~dB}$ using this frequency instead of $4 \mathrm{kHz}$. Another example is given in Fig. 3 (c) for the configuration shown in Fig. 2(f), where $r_{0}=1.87$, 2.81 , and $3.74 \mathrm{~km}$ for $D=10,15$, and $20 \mathrm{~km}$, respectively. One finds for $D=10 \mathrm{~km}$, the optimum frequency for this configuration is $f \sim 20 \mathrm{kHz}$. One gains a saving of $\sim 8.6 \mathrm{~dB}$ using this frequency instead of $4 \mathrm{kHz}$. For $D=15 \mathrm{~km}$, the optimum frequency for this configuration is $f \sim 16 \mathrm{kHz}$. One gains a saving of $\sim 6.9 \mathrm{~dB}$ using this frequency instead of $4 \mathrm{kHz}$. For $D=20 \mathrm{~km}$, the optimum frequency for this configuration is $f \sim 14 \mathrm{kHz}$. One gains a saving of $\sim 5.8 \mathrm{~dB}$ using this frequency instead of $4 \mathrm{kHz}$. These examples show that for each configuration, one can determine the optimal frequency given an area of coverage (or the diameter). Two comments are in order here. First, the optimum frequency for a given configuration changes somewhat depending on the value of $k$ and $\alpha$ used. Hence, for a given area of operation, one needs to apply a propagation model to determine the appropriate values to use. Second, given an (optimum) frequency, say, $16 \mathrm{kHz}$, one can generate a total power figure, similar to Fig. 3(a). One would find that the conventional tomographic approach with sensors spread on the periphery uses much more power than the distributed sensors approach and the total power for distributed sensors decreases rapidly with an increasing number of sensors.

Given a sensor configuration/topology, one can calculate the optimum frequency as shown in Figs. 3(b) and 3(c). Alternatively, one can calculate the optimum frequency (from the prospective of total energy), if the sensor separation is known. Noting that $P_{N}\left(r_{0} ; f\right)-P_{N}\left(r_{0} ; f_{0}\right)=P\left(r_{0} ; f\right)$ $-P\left(r_{0}: f_{0}\right)$ is a function of $r_{0}$ and $f$, one can display this difference in terms of a two-dimensional surface in a color plot as shown in Fig. 4. One finds that the optimal frequency is a function of the node distance as shown by the dots in Fig. 4. It indicates that the shorter the node separation, the higher the optimum frequency to use. This result agrees with the rule of thumb used in underwater acoustic communications where the carrier frequency is inversely proportional to the square root of the distance.

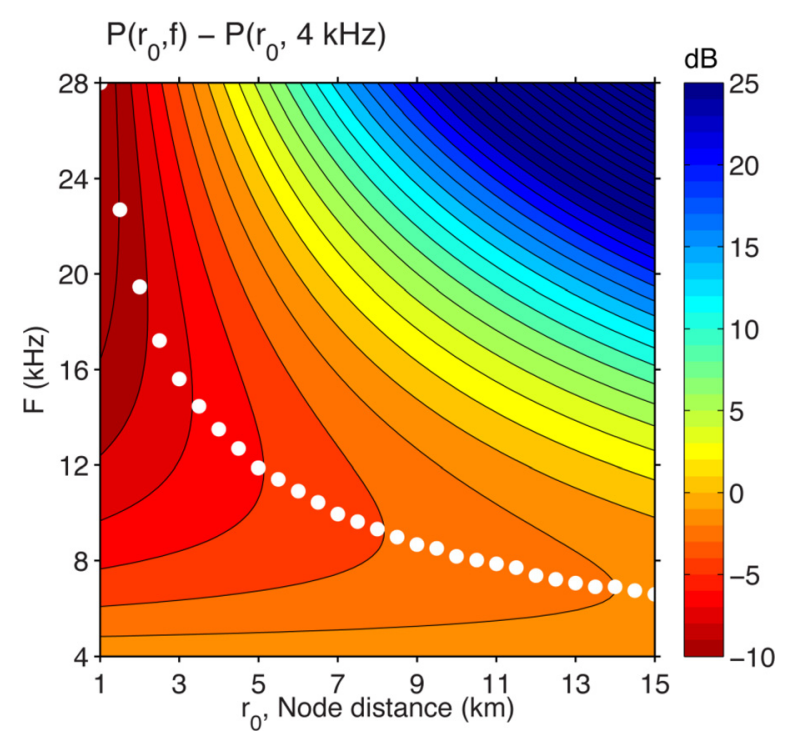

FIG. 4. (Color online) The transmission power as a function of frequency and node separation with respect to that of the $4 \mathrm{kHz}$ system.

In conclusion, one finds that DNUS systems generally use much less power (as much as $15 \mathrm{~dB}$ ) in signal projection compared with the conventional OAT system implying many-fold extension in a lifetime for battery-powered systems. At the same time, a similar (or better) performance to that of the OAT system can achieved by increasing (doubling) the number of sensors (without additional cost in power) as illustrated by the examples in Sec. IV.

\section{PERFORMANCE COMPARISON USING TOMOGRAPHIC INVERSION}

For a conventional OAT system, given $N$ sensors, there are $N(N-1) / 2$ independent input data. The system is equivalent to a number of virtual nodes, on the order of $N^{2}$, for sampling the current field. The distributed systems use physical nodes to sample the current field. For the sensor configurations in Figs. 2(b)-2(f), there are $M$ triangles providing $M$ independent measurements of the current in each triangle, where $M$ is a function of the number of sensor nodes in each system. Thus, it is expected that for a large enough $M$, the system should provide an equal performance as the conventional OAT system based on the spatial sampling theorem. Note that data from physical nodes are free of extrapolation errors versus data using virtual nodes. The performances of OAT and DNUS systems are studied in this section using simulated data for different current fields under different model assumptions. The results provide an idea of how many distributed sensors are needed to achieve the same performance as an OAT system.

\section{A. Basic equations}

Assuming a constant sound speed and current field between two points, one can measure the current along the propagation path by the travel time difference between reciprocal sound transmissions. The one-way travel times $t_{12}$ and $t_{21}$ can be expressed as 


$$
\begin{aligned}
& t_{12}=\frac{R}{c+\mathbf{v} \cdot \mathbf{u}_{12}} \simeq \frac{R}{c}\left(1-\frac{\mathbf{v} \cdot \mathbf{u}_{12}}{c}\right), \\
& t_{21}=\frac{R}{c+\mathbf{v} \cdot \mathbf{u}_{21}} \simeq \frac{R}{c}\left(1-\frac{\mathbf{v} \cdot \mathbf{u}_{21}}{c}\right),
\end{aligned}
$$

where $\mathbf{v}$ is the current vector and $\mathbf{u}_{12}$ is a unit vector along the acoustic path from point 1 to point 2 . The sound speed and current projection along the ray path can be estimated from the mean travel time $T_{12}=\left(t_{12}+t_{21}\right) / 2$ and the travel time difference $\Delta t_{12} \equiv\left(t_{12}-t_{21}\right) / 2$ as follows:

$$
\begin{aligned}
& c=\frac{R}{T_{12}} \\
& \mathbf{v} \cdot \mathbf{u}_{12}=-\frac{\Delta t_{12} R}{T_{12}^{2}} .
\end{aligned}
$$

For a non-uniform current field, the travel time difference between sensors $i$ and $j$, divided by 2 , is given by a line integral of the current projected along the path, divided by the sound speed squared

$$
\Delta t_{i j}=-\int \frac{\mathbf{v}(s) \cdot \mathbf{u}_{i j}}{c^{2}(s)} d s .
$$

Equation (6) forms the basis for current inversion.

Recent tomographic inversion algorithms often express the unknown (current) field in terms of a two-dimensional wavenumber spectrum and invert for the spectral coefficients using Eq. (6). Having estimated the wavenumber spectrum, one obtains an estimate of the original field by an inverse Fourier transform. For the current inversion, it is convenient to define a stream function ${ }^{13} \psi$ from which one can derive the current field using $\mathbf{v}=-\nabla \times \psi$. Using a truncated Fourier series representation for the stream function $\psi(x, y)$,

$$
\begin{aligned}
\psi(x, y)= & \sum_{k=0}^{K_{\max }-1} \sum_{l=-l^{\prime}(k)}^{l^{\prime}(k)}\left[A_{k l} \cos \left(\frac{2 \pi k x}{L_{x}}+\frac{2 \pi l y}{L_{y}}\right)\right. \\
& \left.+B_{k l} \sin \left(\frac{2 \pi k x}{L_{x}}+\frac{2 \pi l y}{L_{y}}\right)\right],
\end{aligned}
$$

where $l^{\prime}(k)=\left(K_{\max }^{2}\right)^{1 / 2} ; 2 \pi\left(K_{\max }-1\right) / L_{x}$ is the maximum wavenumber for the current field; $\left[A_{k l}, B_{k l}\right]$ are the Fourier coefficients; and $L_{x}$ and $L_{y}$ are the length parameters which need to be chosen to be (significantly) greater than $D$, since the model is periodic with respect to $L_{x}$ and $L_{y} . K_{\max }$ sets the nominal spatial resolution of the inversion model which is on the order of $\left[L_{x} / K_{\max }, L_{y} / K_{\max }\right]$.

Using Eqs. (6) and (7), one can express the travel time difference data $\mathbf{d}$ as a function of the Fourier coefficients $\mathbf{m}=[\mathbf{A} ; \mathbf{B}]$ in a matrix form as follows:

$$
\mathbf{d}=\mathbf{G m}+\mathbf{n},
$$

where $\mathbf{A}$ and $\mathbf{B}$ are the vectors containing all the Fourier coefficients $\left[A_{k l}, B_{k l}\right]$. The objective is to solve for the Fourier coefficients from which one can obtain the stream function and correspondingly the current field distribution.
When the number of (unknown) Fourier coefficients is larger than the number of input data points, one has an underdetermined system. To solve an underdetermined system, one imposes a regulator and solves the equation by minimizing the following objective function:

$$
J(\mathbf{m})=(\mathbf{d}-\mathbf{G m})^{T} \mathbf{R}^{-1}(\mathbf{d}-\mathbf{G m})+\beta \mathbf{m}^{T} \mathbf{S}^{-1} \mathbf{m},
$$

where $\mathbf{R}$ is the covariance matrix of the (measurement) noise, and $\mathbf{S}$ is the regulator matrix. ${ }^{2}$ Although $\beta$ can be absorbed into either $\mathbf{R}$ or $\mathbf{S}$, it is reserved here for separate control. Assuming both matrices are positive definite and symmetric, they have Cholesky decompositions. In terms of the square root of the matrices defined below

$$
\begin{aligned}
\mathbf{G}^{\prime} & =\mathbf{R}^{-T / 2} \mathbf{G} \mathbf{S}^{1 / 2}, \mathbf{m}^{\prime}=\mathbf{S}^{-\mathrm{T} / 2} \mathbf{m}, \mathbf{n}^{\prime}=\mathbf{R}^{-T / 2} \mathbf{n}, \\
\mathbf{d}^{\prime} & =\mathbf{R}^{-\mathrm{T} / 2} \mathbf{d},
\end{aligned}
$$

one can transform the weighted $l_{2}$ inverse problem, Eq. (9), into a simple equation

$$
J\left(\mathbf{m}^{\prime}\right)=\left(\mathbf{d}^{\prime}-\mathbf{G}^{\prime} \mathbf{m}^{\prime}\right)^{T}\left(\mathbf{d}^{\prime}-\mathbf{G}^{\prime} \mathbf{m}^{\prime}\right)+\beta \mathbf{m}^{\prime T} \mathbf{m}^{\prime},
$$

whose solution can be written in an analytic form as follows: ${ }^{2}$

$$
\hat{\mathbf{m}}^{\prime}=\left(\mathbf{G}^{\prime T} \mathbf{G}^{\prime}+\beta \mathbf{I}\right)^{-1} \mathbf{G}^{\prime T} \mathbf{d}^{\prime}
$$

where the "hat" above a symbol indicates an estimator of the variable. The parameter $\beta$ will be determined using the $L$ curve criterion in the transformed problem. ${ }^{14}$ The original field is then given by

$$
\begin{aligned}
\hat{\mathbf{m}} & =\mathbf{S}^{1 / 2} \hat{\mathbf{m}}^{\prime} \\
& =\mathbf{S}^{T / 2}\left(\mathbf{S}^{T / 2} \mathbf{G}^{T} \mathbf{R}^{-1} \mathbf{G} \mathbf{S}^{1 / 2}+\beta \mathbf{I}\right)^{-1} \mathbf{S}^{T / 2} \mathbf{G}^{T} \mathbf{R}^{-1} \mathbf{d}
\end{aligned}
$$

The model solution $\hat{\mathbf{m}}$ is the Fourier coefficients of the stream function and is mapped to physical space for the current fields using a matrix transform $\hat{\mathbf{v}}(x, y)=\mathbf{T} \hat{\mathbf{m}}$ where the columns of $\mathbf{T}$ are the Fourier function expression of the current field used in the inversion.

One notes that the above solution is dependent on the model weight matrix $\mathbf{S}$, which is usually determined by assuming some prior knowledge about the (current) field; the better the prior knowledge agrees with the truth, the more accurate the solution will be. In our case the unknown (model) parameters are the Fourier coefficients of the stream function and the model weight matrix $\mathbf{S}$ specifies some prior knowledge about the wavenumber spectrum.

We shall assume a diagonal $\mathbf{S}$ whose $i$ th entry is proportional to $\left(\left|\mathbf{k}_{i}\right|^{2}+k_{0}^{2}\right)^{-3 / 2}$ where $k_{0}=2 \pi / X_{0}$. The length scale $X_{0}$ sets the "roll over" wavenumber of the spectrum. For numerical studies, we shall use $X_{0}=1,5$, and $10 \mathrm{~km}$. One notes that higher $X_{0}$ puts more weight on the lower wavenumbers. For example, using $X_{0}=10 \mathrm{~km}$, high wavenumber components above $0.6 \mathrm{~km}^{-1}$ are suppressed by $e^{-1}$ or more. Thus, if one has some prior knowledge that the wavenumber 
spectrum is concentrated at low wavenumbers (correlation length $<1 \mathrm{~km}$ ), one can expect a better inversion result by using $X_{0}=10 \mathrm{~km}$. On the other hand, without having any knowledge about the spectrum, assuming $X_{0}=1 \mathrm{~km}$ produces a nearly equal weighting on all wavenumbers.

For the (measurement) noise covariance matrix, we assume

$$
\mathbf{R}=\operatorname{diag}\left[\sigma_{t}^{2}+\left(\frac{s_{i j} \sigma_{u}}{c^{2}}\right)^{2}\right],
$$

where the first term, $\sigma_{i}^{2}$, denotes mean-square travel time errors due to the finite signal $\mathrm{BW}$, and the second term denotes the mean-square travel time errors due to medium fluctuations (induced by scattering from medium inhomogeneities), which is proportional to the path length; $s_{i j}$ denotes the path length between $i$ and $j$ stations and $\sigma_{u}$ denotes the current standard deviation. ${ }^{5}$

For performance evaluations of various approaches, we shall use the root-mean-square error (RMSE) between the estimated and original velocity magnitudes $|\mathbf{v}|=\sqrt{u^{2}+v^{2}}$ in this paper, which is defined by

$$
\operatorname{RMSE}=\sqrt{\frac{1}{J} \sum_{j=1}^{J}\left(|\mathbf{v}|_{j}-|\hat{\mathbf{v}}|_{j}\right)^{2}},
$$

where the subscript $j$ indicates current field inverted at the $j$ th cell (position) in the physical space. We also study the estimation error in the physical domain $(x, y)$ in terms of the difference between the estimated and true current magnitude $\delta|\mathbf{v}|=|\hat{\mathbf{v}}|-|\mathbf{v}|$, and in the wavenumber domain $\left(k_{x}, k_{y}\right)$ in terms of the error spectrum, i.e., the difference of the spectrum between the estimated and true current magnitudes $\delta|\mathbf{V}|=|\hat{\mathbf{V}}|-|\mathbf{V}|$. One also defines the fractional residual error variance of the velocity magnitude

$$
\sigma_{|\mathbf{V}|}^{2}=\frac{\left\langle(\delta|\mathbf{V}|)^{2}\right\rangle}{\left\langle|\mathbf{V}|^{2}\right\rangle}=\frac{\left\langle(\delta|\mathbf{v}|)^{2}\right\rangle}{\left\langle|\mathbf{v}|^{2}\right\rangle}=\sigma_{|\mathbf{v}|}^{2},
$$

where the average \langle\rangle is over the wavenumbers or the spatial coordinates. Note that Eqs. (15) and (16) are calculated with respect to one (given) true field. For each selected sensor configuration, RMSE and $\sigma_{|\mathbf{v}|}^{2}$ are calculated using different $X_{0}$. The results are presented in Sec. IV B.

\section{B. Simulation results}

This section shows the inversion results using the tomographic inversion method for the OAT system [Fig. 2(a)] and DNUS systems [Figs. 2(b) and 2(c)] based on simulated data. How the current field is simulated is discussed next. For the simulated data, the current velocities are timeinvariant.

To generate the current field, one defines a stream function $\psi$, which has a power spectrum composed of a set of Gaussian functions given by

$$
P(\mathbf{k})=\sum_{j=1}^{4} W_{j} \exp \left[-\frac{\left(\mathbf{K}_{j}-\mathbf{k}\right)^{2}}{2 K_{0}^{2}}\right]
$$

The current is obtained by $\mathbf{v}=-\nabla \times \psi$. We consider two cases: Narrowband and broadband spectrum cases, as shown in Figs. 5(a-1) and 5(b-1), respectively. For the narrowband case, for example, the complex wavenumbers are given by $\left|\mathbf{K}_{j}\right|=[2 \pi / 5.8,2 \pi / 6.3,2 \pi / 7.2,2 \pi / 7.4]\left(\mathrm{km}^{-1}\right)$, and phases, $\arg \left(\mathbf{K}_{j}\right)=\left[10^{\circ}, 0^{\circ}, 20^{\circ},-20^{\circ}\right]$. The Gaussian distributions have a standard deviation $K_{0}=0.05 \sum_{j=1}^{4} K_{j}$, and are weighted by $W_{j}=[1,0.7,0.4,0.8]$. The spectrum has a mean wavelength of approximately $6.7 \mathrm{~km}$ and have a main axis oriented $\sim 5^{\circ}$ from the $y$ axis. The bottom panels of Fig. 5 show the true current fields obtained by the stream functions constructed by using the power spectra [Eq. (17)] for amplitudes and uniformly distributed random numbers for phases. For the broadband case, the power spectrum is isotropic and has a correlation length of $0.7 \mathrm{~km}$, resulting in a relatively small scale rotary motion compared to the narrowband case.

Given the simulated current field, one creates the synthetic data for travel time differences between all pairs of nodes for two sensor topologies [Figs. 2(a) and 2(b)], with a diameter $D=5 \mathrm{~km}$, assuming a sound speed of $1500 \mathrm{~m} / \mathrm{s}$. This will be the input data to test the tomographic algorithm and to compare how the inversion results vary with the sensor topology. For convenience, we first consider the following cases of ray distributions sampled by the sensor topologies shown in Figs. 2(a) and 2(b):

Case I: The 8-node conventional OAT system where the rays connect all pairs of nodes in Fig. 2(a). It contains 28 lines or 28 input data points for the travel time differences.
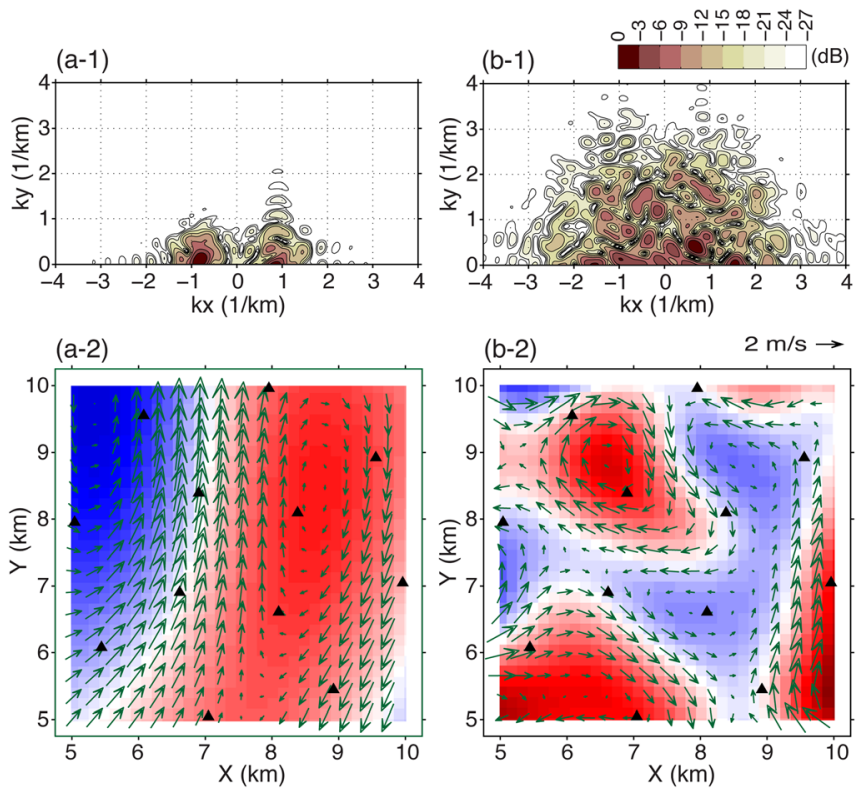

FIG. 5. (Color online) Wavenumber spectra of stream function (top) used to generate a simulated current field (bottom) for (a) narrowband and (b) broadband cases. The magnitude of the stream function is indicated by the color. The derived current field is shown by the arrows at various positions. The triangle symbols denote the sensor locations. 
Case II: The 12-node DNUS system where the rays connect only the nearest neighboring nodes (short rays) in Fig. 2(b). There are 25 lines or 25 (independent) travel time difference data.

Case III: The 12-node system where the rays connect all (possible) pairs of nodes in Fig. 2(b) (including both the short and long rays). This will be a 12-node OAT system. There will be 66 lines connecting different nodes but in this case some of the lines overlap the others due to symmetric placement of the inner nodes with respect to the outer nodes. All 66 travel time differences are used in the inversion.

The results of the tomographic inversion are summarized in Fig. 6. The noise covariance is assumed a an identity matrix. The left panel in Fig. 6 shows the inversion results for the narrowband current field using different $X_{0}$ 's. One finds that the inversion results for case I (the 8-node conventional OAT system; denoted by square symbols) yield an overall residual variance $\sigma_{|\mathbf{v}|}^{2}=3.31 \%$ using a nearly flat model weight $\left(X_{0}=1 \mathrm{~km}\right)$. As more weights are imposed on the lower wavenumbers $\left(X_{0}=5,10 \mathrm{~km}\right)$, the error variances are reduced. This agrees with the narrowband nature of the current field. Inversions from case II (the 12-node DNUS system with short rays; denoted by circles) show a little less error variance reduction than those from case I. For case III (the 12-node OAT system with both short and long rays; denoted by triangles), the error variance diminishes to an average of $0.3 \%$. This represents a significant improvement compared with case I since many more rays are presented in this case ( 66 for case III vs 28 for case I). The right panel in Fig. 6 shows the inversion results for a broadband current field. The open and solid symbols indicate the results with a maximum wavenumber of $2.2 \mathrm{~km}^{-1} \quad\left(K_{\max }=8\right)$ and $3.5 \mathrm{~km}^{-1}\left(K_{\max }=12\right)$, respectively. When the maximum wavenumber of the stream function used in the model is larger than the maximum wavenumber of the true field, the overall inversion performance is generally improved (smaller error variance).

To understand the results, we examine the inversion results in details, as shown in Fig. 7 for the narrowband case (using $K_{\max }=8$ ). The top three rows show the results (from (a) Narrowband

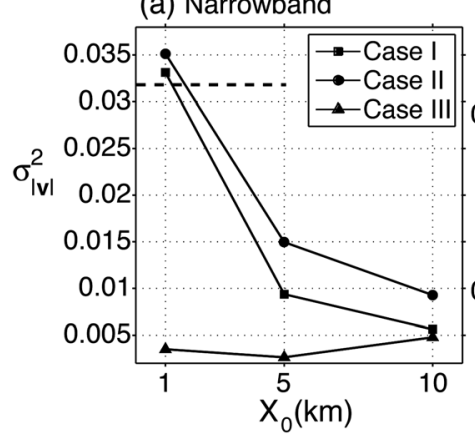

(b) Broadband

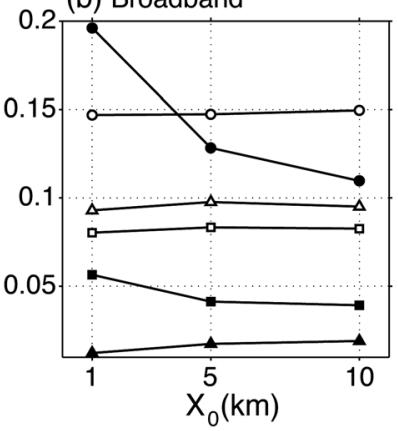

FIG. 6. Fractional residual error variance of the current magnitude $\sigma_{\mid \mathrm{v}}^{2}$ as a function of the rollover length $X_{0}$. The lower value in error variance indicates the estimated field is closer to the original field. The square, circle, and triangle symbols denote different array designs: Cases I, II, and III, respectively. The horizontal dashed line in (a) indicates the inversion using the distributed signal processing algorithm. top to bottom) for cases I, II, and III, respectively. The two vertical columns correspond to $X_{0}=1$ and $10 \mathrm{~km}$, respectively. For each case with a given $X_{0}$, there are two figures: The left figure shows the estimation error of the current magnitudes in the physical space, with the RMSE displayed on the top, and the right figure shows the error spectrum (normalized by the maximum of the original spectrum) and the fractional residual error variance displayed on the top of the figure. One observes that the error spectra are similar between the 8-node OAT system (case I) and the 12-node DNUS system (case II), both decreasing with increasing $X_{0}$, with case I having a somewhat smaller fractional residual error variance. Case III (12 nodes using both short and long rays) shows a much smaller ( $>10 \mathrm{~dB})$ error spectrum and an order of magnitude reduction in the fractional residual error variance. In this case, the wavenumber spectrum is better resolved by using both short and long rays. In all events, one finds that a simple model which minimizes the model norm $\mathbf{m}^{T} \mathbf{m}\left(\mathbf{S} \cong \mathbf{I}\right.$ for $\left.X_{0}=1 \mathrm{~km}\right)$ is insufficient to stabilize the estimate, since it has a tendency to spread the "energy" over all (allowed) wavenumbers. ${ }^{15}$

One also estimates the current field for the broadband case and calculates the error spectra for the various configurations. The details are not shown here, but some discussions of the results presented in Fig. 6(b) are in order. It is noted that if the model uses too small a $K_{\max }$, the truncation artifacts may limit the ability to fit the data. ${ }^{15,16}$ For the present case, some high wavenumber components of the current field may have been truncated using $K_{\max }=8$, thus ending up with a poor estimate of the current field [open symbols in Fig. 6(b)]. One finds that the model smoothness constraint imposed by $X_{0}$ plays an insignificant role in the results. On the other hand, the system becomes underdetermined using a high $K_{\max }\left(K_{\max }=12\right.$ introduces 1.5 times more Fourier coefficients than $K_{\max }=8$ ) and is soluble only with additional constraints. For cases I and III, model smoothness in terms of $X_{0}=10 \mathrm{~km}$ seems to improve resolvability of the relatively low wavenumber components. In fact, one finds that the weighted model spectrum using $X_{0}=10 \mathrm{~km}$ (not shown here) is comparable to the original spectrum averaged over all azimuth angles, noting that the original spectrum is peaked at a low wavenumber. For case II, one finds a larger fractional error variance using $K_{\max }=12$ than 8 , suggesting that some degrees of freedom are not properly constrained; note that the sampling density of rays (number of passing rays per unit area) is less for case II than for cases I and III. The above results are consistent with the general findings that for tomographic inversion, a proper choice of the regulator and $K_{\max }$ can significantly improve the inversion results.

To obtain improved results, an alternative method is to increase the number of sensors using a distributed system. We will show that the benefit of adding four more sensors (from 12 nodes to 16 nodes) more than compensates the lack of knowledge on the regulator.

Figure 8 shows the inversion results using the tomographic inversion algorithm for the 16-node DNUS system [Fig. 2(c)] using only short rays. The top row shows the inversion result for the narrowband case and the bottom row shows the inversion result for the broadband case to compare with 
$X_{0}=1 \mathrm{~km}$
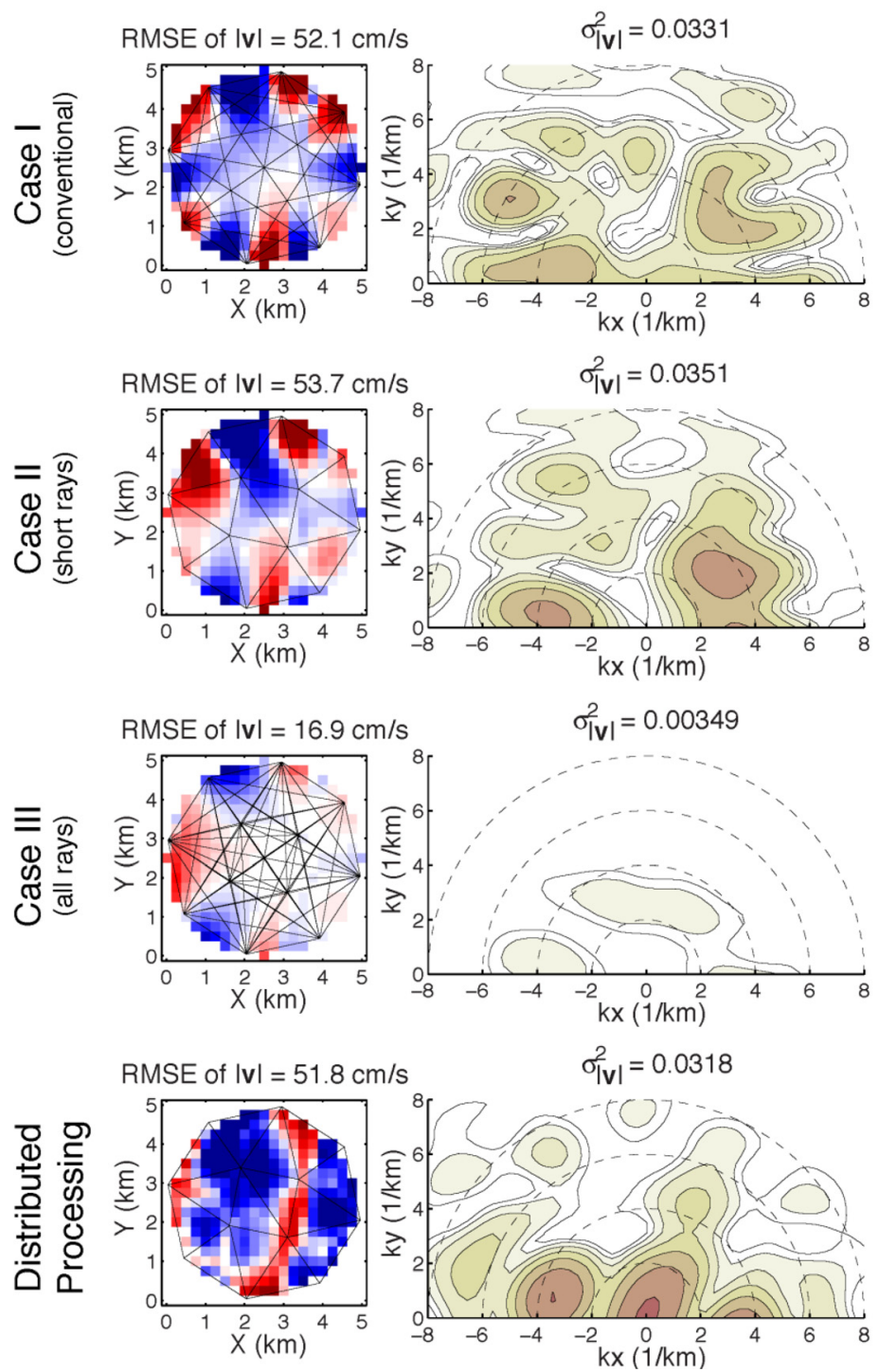
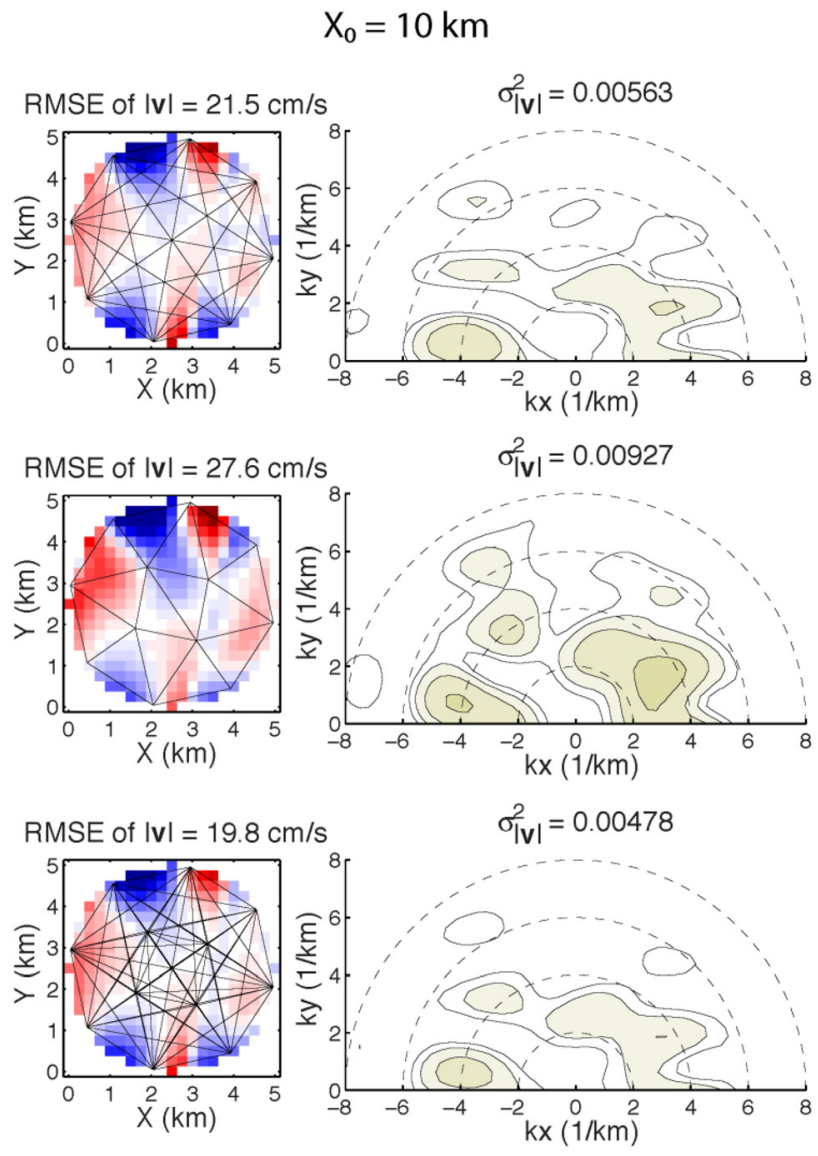

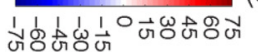

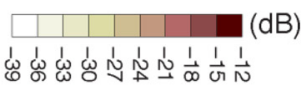

FIG. 7. (Color online) The top three row panels show the inversion results for cases I, II, and III with different values of $X_{0}$. The two vertical columns are for $X_{0}=1$ and $10 \mathrm{~km}$. For each case, the left panel shows the estimation error of the current magnitude in the physical domain and the right panel shows the normalized error spectrum. The color bar for all spectral images has a dynamic range of $27 \mathrm{~dB}$.

that shown in Fig. 7. In both cases, one assumes no prior knowledge about the current field, using the model weight spectrum with $X_{0}=1 \mathrm{~km}$. One finds that the estimated current fields from the 16-node DNUS with short rays [Fig. 2(c)] yields a much small fractional error variance compared to the conventional OAT system [Fig. 2(a)]. For the narrowband case, the fractional error variance is reduced to $0.0117 \%$ for the 16-node DNUS system compared with the best case results of $0.563 \%$ variation for the 8-node OAT system (a factor of $\sim 48$ improvement). For the broadband case, the fractional error variance is $0.712 \%$ for the 16-nodes DNUS system compared with $3.92 \%$ of the original 8-node OAT (a factor of $\sim 5.5$ improvement). The results suggest that distributed systems can sample the low as well as the high wavenumber parts of the spectrum using just the short-ray data given a sufficient number of nodes. In the present case, the DNUS system using a doubled number of nodes (16) yields a much better result than the 8-node OAT system.

\section{DISTRIBUTED SIGNAL PROCESSING}

While the tomographic inversion algorithm can be applied to the DNUS systems, it is generally computationally intensive. For practical purposes, to allow in-buoy processing and real-time telemetry of the inversion results, we propose a simple distributed signal processing for estimating the current field with a slight degradation in the performance which can be easily compensated with an increase in the number of nodes used. The algorithm is applied to each triangle in the DNUS system, for which we give an error analysis, which shows that minimum error is obtained when none of the angles is small.

\section{A. Error analysis}

To determine the current vector $\mathbf{v}$, one needs current measurements along two paths. Let $\mathbf{u}_{12}$ and $\mathbf{u}_{13}$ be the unit vectors from points 1 to 2 and points 1 to 3 , respectively 

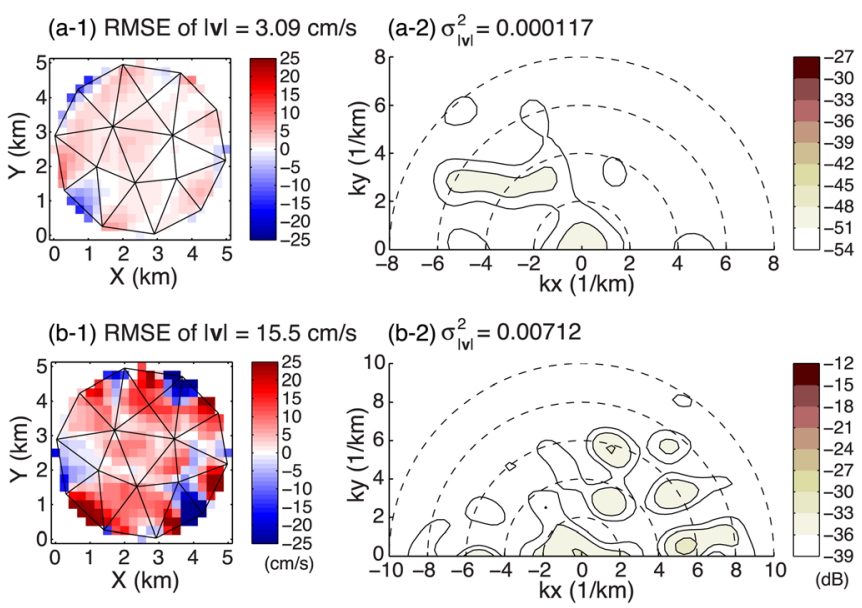

FIG. 8. (Color online) Tomographic inversion results for the 16-node DNUS system [with short rays, see Fig. 2(c)] for the narrowband (a) and broadband (b) current fields. In both cases, one uses $X_{0}=1 \mathrm{~km}$, i.e., no prior knowledge of the field.

[see, for example, Fig. 9(b)]. Let the angle between the two unit vectors be denoted as $\theta$. We will study the uncertainty in the current estimate as a function of $\theta$ due to errors in travel time difference measurements (in real data). For this purpose, we shall express the travel time as the sum of the mean plus a fluctuation term, given by $\Delta t_{12}=\overline{\Delta t}_{12}+\delta_{12}$ and $\Delta t_{13}=\overline{\Delta t}_{13}$ $+\delta_{13}$ where $\delta_{12}$ and $\delta_{13}$ are assumed to be two independent (Gaussian) random variables with a standard deviation $\sigma$ determined by the signal $\mathrm{BW}$ and the travel time fluctuations induced by scattering from medium inhomogeneities.

Let $\mathbf{u}_{L}$ denote the longitudinal unit vector along the bisector of the angle between $\mathbf{u}_{12}$ and $\mathbf{u}_{13}$, and $\nu_{L}=\mathbf{v} \cdot \mathbf{u}_{L}$ be the current projection along the longitudinal axis. Let $\mathbf{u}_{\perp}$ be the unit vector perpendicular to $\mathbf{u}_{L}$, and $v_{\perp}=\mathbf{v} \cdot \mathbf{u}_{\perp}$ be the current projection along the transverse axis. With some calculations, one finds that $\delta v_{L}=-0.5\left(R_{12} \delta_{12} / T_{12}^{2}+R_{13} \delta_{13} / T_{13}^{2}\right) / \cos (\theta / 2)$ and $\delta v_{\perp}=-0.5\left(R_{12} \delta_{12} / T_{12}^{2}-R_{13} \delta_{13} / T_{13}^{2}\right) / \sin (\theta / 2)$, with a standard deviation given by $\sigma_{L}=\gamma / \cos (\theta / 2)$ and $\sigma_{\perp}=\gamma / \sin (\theta /$ 2), respectively, where $\gamma=0.5 \sigma \sqrt{R_{12}^{2} / T_{12}^{4}+R_{13}^{2} / T_{13}^{4}} ; R$ and $T$ denote the true range and mean travel time, respectively. These results show that the transverse current measurement has a large error when the angle $\theta$ between the two vectors is small (e.g., $\theta<20^{\circ}$ ), and the longitudinal current measurement has a large error when the angle $\theta$ between the two vectors is large (e.g., $\theta>160^{\circ}$ ). As expected, when the two vectors are orthogonal the solution with the minimum error is obtained for both the longitudinal and transverse measurements. For a triangle, the optimal solution is the equilateral triangle as remarked in Sec. II.

$$
\mathbf{G}=\frac{-1}{2 c^{2}}\left[\begin{array}{cccccc}
s_{13} \cos \theta_{13} & s_{13} \sin \theta_{13} & 0 & 0 & s_{13} \cos \theta_{13} & s_{13} \sin \theta_{13} \\
s_{12} \cos \theta_{12} & s_{12} \sin \theta_{12} & s_{12} \cos \theta_{12} & s_{12} \sin \theta_{12} & 0 & 0 \\
0 & 0 & s_{23} \cos \theta_{23} & s_{23} \sin \theta_{23} & s_{23} \cos \theta_{23} & s_{23} \sin \theta_{23}
\end{array}\right]
$$

(b) 3 (a)
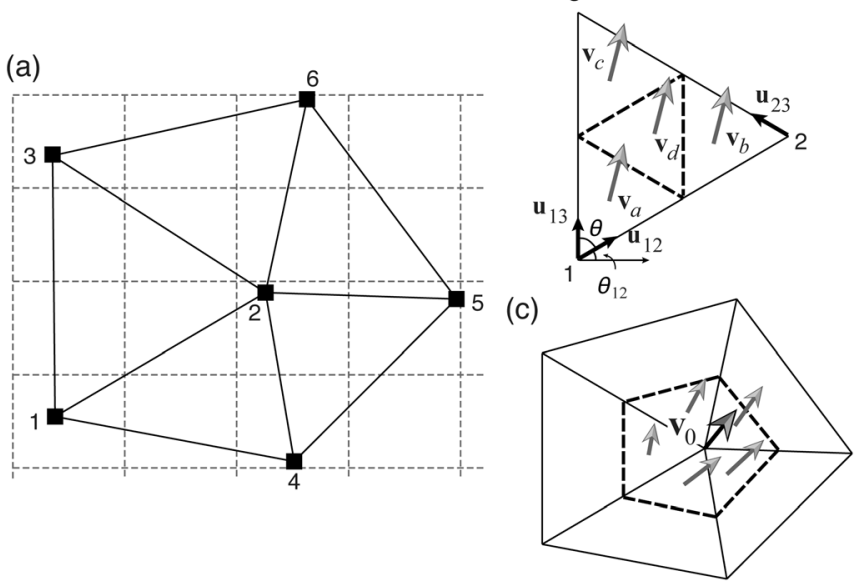

FIG. 9. (a) A distributed system. (b) Distributed sensing of currents for each triangle. (c) Current estimate at the intersection points of many triangles.

\section{B. Current estimation}

For DNUS, the signals are designed to reach only the neighboring nodes. One can group the neighboring nodes into non-overlapping triangles [Figs. 2(b)-2(f)]. One can solve Eq. (8) by solving the sub-matrix equation $\mathbf{d}=\mathbf{G v}+\mathbf{n}$ for each triangle, since the equation is uncoupled between triangles and thus can be solved individually. Here, we use $\mathbf{v}$ to represent the unknown current field. Consider one triangle as shown in Fig. 9(b) with three nodes denoted by 1, 2, and 3. To invert for the current within the triangle, the triangle is divided into four sub-triangles denoted by triangles $\Delta_{a}, \Delta_{b}$, $\Delta_{c}$, and $\Delta_{d}$ in Fig. 9(b). The current vectors $\mathbf{v}_{a}, \mathbf{v}_{b}$, and $\mathbf{v}_{c}$ at the sub-triangles $\Delta_{a}, \Delta_{b}$, and $\Delta_{c}$ are related to the measurement data by (ignoring the noise)

$$
\begin{aligned}
& \Delta t_{13}=\frac{t_{13}-t_{31}}{2}=-\frac{s_{13}}{c^{2}} \frac{\mathbf{v}_{a} \cdot \mathbf{u}_{13}+\mathbf{v}_{c} \cdot \mathbf{u}_{13}}{2} \\
& \Delta t_{12}=\frac{t_{12}-t_{21}}{2}=-\frac{s_{12}}{c^{2}} \frac{\mathbf{v}_{a} \cdot \mathbf{u}_{12}+\mathbf{v}_{b} \cdot \mathbf{u}_{12}}{2} \\
& \Delta t_{23}=\frac{t_{23}-t_{32}}{2}=-\frac{s_{23}}{c^{2}} \frac{\mathbf{v}_{b} \cdot \mathbf{u}_{23}+\mathbf{v}_{c} \cdot \mathbf{u}_{23}}{2},
\end{aligned}
$$

where $s_{12}, s_{13}$, and $s_{23}$ are the side lengths for each triangle, and $\mathbf{u}_{12}, \mathbf{u}_{13}$, and $\mathbf{u}_{23}$ are the unit vectors along the paths from nodes 1 to 2,1 to 3 , and 2 to 3 , respectively [Fig. 9(b)]. The above equation can be written in a matrix form $\mathbf{d}=\mathbf{G v}$, where $\mathbf{d}=\left[\Delta t_{13}, \Delta t_{12}, \Delta t_{23}\right]^{T}$ is the data vector, $\mathbf{v}=\left[\nu_{x, a}, \nu_{y, a}, \nu_{x, b}, \nu_{y, b}, \nu_{x, c}, \nu_{y, c}\right]^{T}$ is the current vector, and 
where one expresses $\mathbf{u}_{i j}=\left[\cos \theta_{i j}\right.$, $\left.\sin \theta_{i j}\right]$. One can solve for $\mathbf{v}_{a}=\left[\nu_{x, a}, \nu_{y, a}\right]$ using the following approximation:

$$
\begin{aligned}
\Delta t_{13} & =-\frac{s_{13}}{c^{2}} \frac{\mathbf{v}_{a} \cdot \mathbf{u}_{13}+\mathbf{v}_{c} \cdot \mathbf{u}_{13}}{2} \\
& =-\frac{s_{13}}{c^{2}}\left[\mathbf{v}_{a} \cdot \mathbf{u}_{13}+\frac{1}{2}\left(\mathbf{v}_{c}-\mathbf{v}_{a}\right) \cdot \mathbf{u}_{13}\right] \\
& \simeq-\frac{s_{13}}{c^{2}}\left(v_{x, a} \cos \theta_{13}+v_{y, a} \sin \theta_{13}\right) \\
\Delta t_{12} & =-\frac{s_{12}}{c^{2}} \frac{\mathbf{v}_{a} \cdot \mathbf{u}_{12}+\mathbf{v}_{b} \cdot \mathbf{u}_{12}}{2} \\
& =-\frac{s_{12}}{c^{2}}\left[\mathbf{v}_{a} \cdot \mathbf{u}_{12}+\frac{1}{2}\left(\mathbf{v}_{b}-\mathbf{v}_{a}\right) \cdot \mathbf{u}_{12}\right] \\
& \simeq-\frac{s_{12}}{c^{2}}\left(v_{x, a} \cos \theta_{12}+v_{y, a} \sin \theta_{12}\right),
\end{aligned}
$$

where we have assumed that the (spatial) variation of the current within the triangle is much smaller than the current itself (which is not a bad assumption if the triangle is not too big), namely, $\left|\mathbf{v}_{c}-\mathbf{v}_{a}\right| \ll\left|\mathbf{v}_{a}\right|$ and $\left|\mathbf{v}_{b}-\mathbf{v}_{a}\right| \ll\left|\mathbf{v}_{a}\right|$, and can thus be neglected. Solving Eq. (20) for $\left[\nu_{x, a}, \nu_{y, a}\right]$, one finds

$$
\begin{aligned}
& \bar{v}_{x, a}=\frac{c^{2}\left(s_{13} \sin \theta_{13} \Delta t_{12}-s_{12} \sin \theta_{12} \Delta t_{13}\right)}{s_{12} s_{13} \sin \left(\theta_{12}-\theta_{13}\right)} \\
& \bar{v}_{y, a}=\frac{c^{2}\left(s_{12} \cos \theta_{12} \Delta t_{13}-s_{13} \cos \theta_{13} \Delta t_{12}\right)}{s_{12} s_{13} \sin \left(\theta_{12}-\theta_{13}\right)},
\end{aligned}
$$

where the overhead "bar" indicates that it is an estimate of the variable. The above analytic solution can be implemented at sensor node 1 to determine the current vector in the sub-triangle $a$. Likewise one can solve for $\overline{\mathbf{v}}_{b}$ $=\left[\bar{v}_{x, b}, \bar{v}_{y, b}\right]$ and $\overline{\mathbf{v}}_{c}=\left[\bar{v}_{x, c}, \bar{v}_{y, c}\right]$ in a similar manner. The current vectors in sub-triangles are assigned at the centroids of sub-triangles. For distributed processing, one does not need the regularization term.

Assuming linear interpolation, the current vector in the middle sub-triangle is given by

$$
\overline{\mathbf{v}}_{d}=\frac{\overline{\mathbf{v}}_{a}+\overline{\mathbf{v}}_{b}+\overline{\mathbf{v}}_{c}}{3},
$$

and the current vector at the node where several triangles intersect [Fig. 9(c)] is given by

$$
\overline{\mathbf{v}}_{0}=\frac{1}{P} \sum_{i=1}^{P} \overline{\mathbf{v}}_{i},
$$

where $\overline{\mathbf{v}}_{i}$ are the estimated current vectors in the neighboring $P$ sub-triangles.

Given the topology of the DNUS system, one determines the sub-triangles, and estimates the current vectors at the centroid of each sub-triangle using Eq. (21), at the middle sub-triangle using Eq. (22) and at the node using Eq. (23). This will be referred to as Method 1. To determine the current field at other locations, one assumes trianglebased linear interpolation between the estimated current vectors using the following formula:

$$
\mathbf{v}(\mathbf{x})=\sum_{k=1}^{3} w_{k}(\mathbf{x}) \overline{\mathbf{v}}_{k},
$$

where $\overline{\mathbf{v}}_{k}$ are the estimated current vectors given above, and $w_{k}(\mathbf{x})$ are the interpolation coefficients.

In many areas, the ocean current field varies slowly spatially (e.g., kilometers), ${ }^{6,7}$ the above approximation may be justified given a small node distance. In other areas, the current may vary significantly within a triangle. The current estimated using the above method for each sub-triangle may be in error since it ignores the variation within each subtriangle; the error is more in the current direction, less so in the current magnitude. In these cases, we ignore the current vectors at the sub-triangles surrounding the inner nodes and keep only the current vectors estimated at the (inner) nodes. The current field is obtained by interpolating the current vectors at the nodes using Eq. (24) as before. This will be referred to as Method 2. The reason is that the current vectors estimated at the inner nodes are more reliable than the current vectors estimated at the neighboring sub-triangles as explained next. (Method 2 keeps part of the data obtained by Method 1.) Consider a node surrounded by many subtriangles as shown in Fig. 9(c). If the sub-triangle configuration is symmetric to the current direction, assuming that the current field varies linearly with space (position), one can show that

$$
\overline{\mathbf{v}}_{0}=\frac{1}{P} \sum_{i=1}^{P} \overline{\mathbf{v}}_{i}=\frac{1}{P} \sum_{i=1}^{P} \mathbf{v}_{i}=\mathbf{v}_{0} .
$$

The reason is as follows. One notices that in Eq. (20), the spatial variation of the current vectors, $\left(\mathbf{v}_{c}-\mathbf{v}_{a}\right) \cdot \mathbf{u}_{13}$ and $\left(\mathbf{v}_{b}-\mathbf{v}_{a}\right) \cdot \mathbf{u}_{12}$ is responsible for the errors in the current estimation. Denoting the central node as node 1 , and summing Eq. (20) over all surrounding sub-triangles, one finds that the summation of $\left(\mathbf{v}_{c}-\mathbf{v}_{a}\right) \cdot \mathbf{u}_{13}$ and $\left(\mathbf{v}_{b}-\mathbf{v}_{a}\right) \cdot \mathbf{u}_{12}$ terms vanishes given the above assumptions. As a result, one obtains Eq. (25). In practice, the sub-triangles will not be symmetric, and there remain some residual errors between $\overline{\mathbf{v}}_{0}$ and $\mathbf{v}_{0}$. For the edge nodes, we keep the current vectors estimated at the sub-triangles in the absence of additional data. As a rule of thumb, the edge nodes should be placed where current variation is small (so that Method 1 is justified) and if in doubt, the current field estimated at the boundary could be dropped.

\section{Simulation results}

\section{Noise free case}

The distributed processing algorithm (Sec. VB) is applied to the various DNUS configurations in Fig. 2 based on the (noise-free) simulated data described in Sec. IV B. For the system in Fig. 2(b), the estimated current field using distributed signal processing and the corresponding error spectrum are shown at the bottom-most panels in Fig. 7 using Method 2. One finds that the $R M S E=51.8 \mathrm{~cm} / \mathrm{s}$, which is comparable to the $\mathrm{RMSE}=53.7 \mathrm{~cm} / \mathrm{s}$ of case II using the tomographic inversion algorithm, assuming no prior knowledge of the current field (i.e., $X_{0}=1 \mathrm{~km}$ ). Distributed processing was also applied to other sensor configurations shown in Figs. 2(d)-2(f). The results are shown 
in Fig. 10 where the fractional error variance is displayed as a function of the number of nodes in each configuration, i.e., $N=12,16,30,42$, and 49 for the DNUS systems shown in Figs. 2(b)-2(f), respectively. For the narrowband current case, Fig. 10(a), one finds that Method 2 yields a smaller fractional error variance than Method 1. For the broadband case, Fig. 10(b), the two methods yield comparable results, except for the 12-node case, where Method 2 yields a higher fractional error variance than Method 1. [One finds that while Method 2 yields a small error variance by averaging over current vectors from neighboring triangles, it sometimes produces a bias, such as when the neighboring triangles are not symmetric with respect to the current field. For the narrowband case, the bias produced by Method 2 is smaller than the error variance produced by Method 1; hence Method 2 is preferred. For the broadband case, the bias produced by Method 2 is comparable and sometimes greater than the variance produced by Method 1 . The reasons have yet to be studied.]

For both methods, one finds that the fractional error variances of the reconstructed current fields decrease with the number of sensors. This is consistent with the earlier observation that improved performance can be obtained by increasing the number of nodes in a distributed system when no prior knowledge of the current field is available.

\section{Noisy data}

In this section, we compare the distributed processing results with the tomographic inversion results in a noisy channel, where the travel time difference fluctuates with time due to sound scattering from sound speed inhomogeneities in the medium. The induced fluctuation increases with the path length. The fluctuation in the travel time difference is assumed to be normally distributed with zero mean and a variance of $1 \%, 5 \%$, or $10 \%$ of the time-difference data. Independent random fluctuations are added to the noise-free synthetic data to produce 300 sets of input data for the travel time differences (between pairs of sensors). Distributed processing and tomographic inversions are applied to each of the simulated data to obtain a fractional error variance. (a)

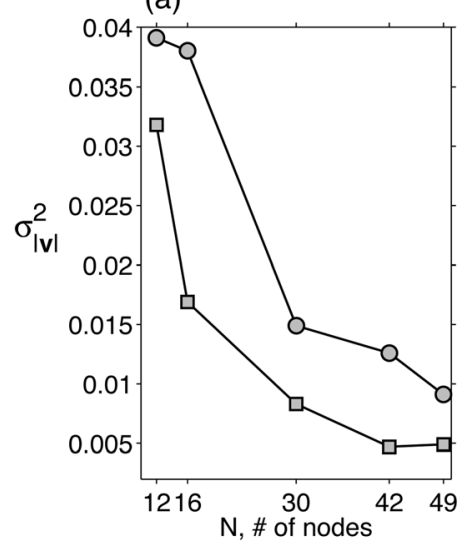

(b)

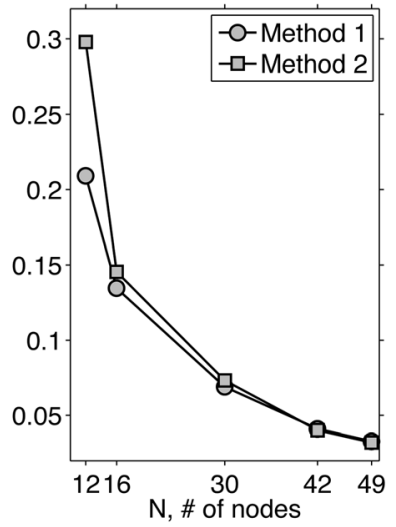

FIG. 10. Fractional residual error variance as a function of the number of nodes using distributed signal processing for (a) narrowband and (b) broadband cases.
The fractional error variance results are averaged over all realizations, and displayed as a function of the percentage of error in the input data as shown in Fig. 11. The top row shows the narrowband results [Figs. 11(a-1) and 11(a-2)] and the bottom row shows the broadband results [Figs. 11(b-1) and 11(b-2)]. The left panel of Fig. 11 shows the inversion results when no prior (model) knowledge is used in the tomographic inversion (e.g., $X_{0}=1 \mathrm{~km}$ ). The right panel of Fig. 11 shows the inversion results using a strong regulator $\left(X_{0}=10 \mathrm{~km}\right)$. We find that distributed processing performs equally well [Fig. 11(b-1)] and sometimes better than the tomographic inversion method [Fig. 11(a-1)] assuming no prior knowledge about the current field $\left(X_{0}=1 \mathrm{~km}\right)$. On the other hand, by incorporating prior knowledge about the current field $\left(X_{0}=10 \mathrm{~km}\right)$, the tomographic inversion yields a much lower fractional error variance than that using distributed processing (which makes no assumption about the field distribution).

To sum up the discussions, the advantage of the distributed sensing algorithm is that it is parameter free and is simple. The only assumption is that the current field varies linearly with position in a local area covering neighboring triangles so that linear interpolation is justified, which is usually valid given reasonable node distances. The disadvantage of distributed processing is that it cannot incorporate prior knowledge to improve the inversion results. In practice, one can set the node distance according to historical data of
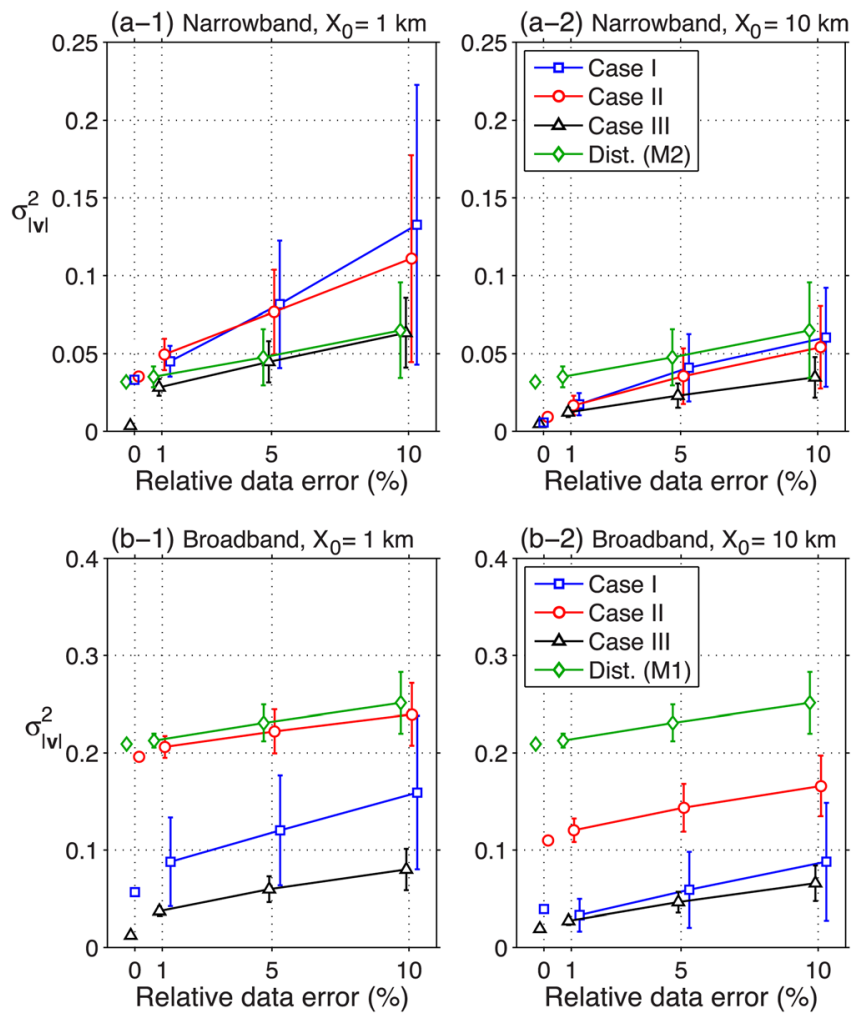

FIG. 11. (Color online) Effect of noise on the residual error variance. Plotted is the fractional residual error variance as a function of the relative error in the differential travel times for (a) narrowband and (b) broadband cases, assuming $X_{0}=1$ and $10 \mathrm{~km}$. The square, circle, and triangle symbols denote different array designs: Cases I, II, and III, respectively. The diamond symbol is for the distributed processing using Method 1 (M1) and Method 2 (M2). 
current gradient, or the desired spatial resolution. The difference in results between Method 1 and Method 2 is expected to decrease; in any case it serves as an indicator for the error size. When in doubt, one can always conduct tomographic inversions for further comparison.

\section{EXPERIMENTAL RESULTS}

An Underwater Networking, Communications, and Acoustic Tomography experiment was conducted near the Sizihwan Bay in Kaohsiung, Taiwan, in May of 2011. It was conducted in an area of $\sim 20 \mathrm{~m}$ depth with the detailed bathymetry and node positions (indicated by the solid circles) shown in Fig. 12. Sixteen conductivity temperature and depth (CTD) measurements were conducted at positions marked by the triangles. The results showed that the sound speed was time varying (by as much as $4 \mathrm{~m} / \mathrm{s}$ during the experiment). A weather buoy equipped with an ADCP was moored in the vicinity of the experimental site (indicated by a square) for continuing hydrological observation of waves and currents.

Each node consists of an acoustic modem moored to the ocean bottom on a rigid platform so that the travel time measurement is not affected by the sensor motion. ${ }^{17}$ The modem is made by Teledyne Benthos (Falmouth, MA) with custom

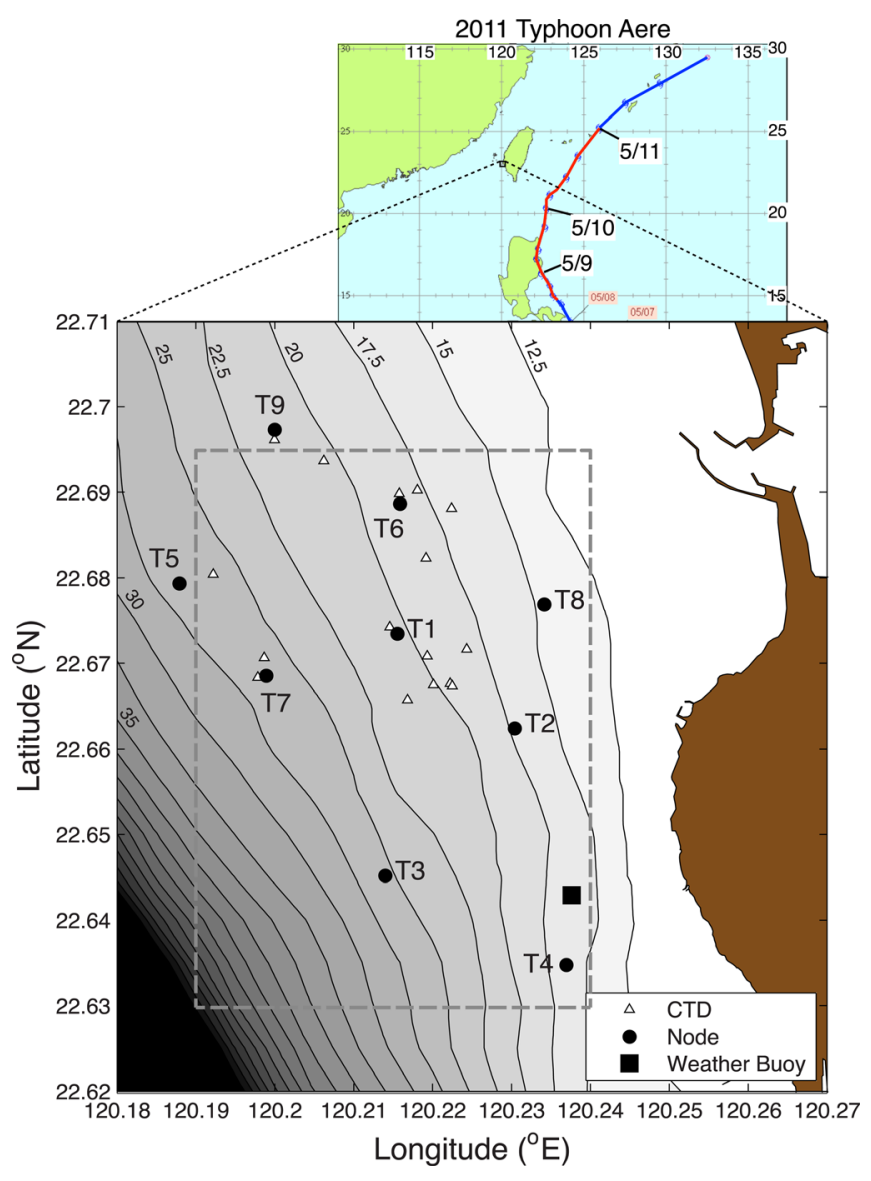

FIG. 12. (Color online) Bathymetry of Marine Test Field with instrument positions (contour interval is $2.5 \mathrm{~m}$ ). Solid circles indicate the acoustic sensor nodes; continuous current measurement was obtained at the weather buoy (square); CTD profiles were obtained at the location indicated by triangles. integrated electronics to transmit and receive arbitrary waveforms. For precise travel time measurements, the modem clock is synchronized to the GPS timing signal via a surface expression. A total of nine nodes were deployed during the experiment. The ranges between neighboring nodes are less than $3 \mathrm{~km}$.

There are 25 tomographic tests during the experiment. During each test, each modem transmits a separately coded $m$-sequence signal modulated by binary phase shift keying, with a carrier frequency of $18.5 \mathrm{kHz}$ and a $\mathrm{BW}$ of $5 \mathrm{kHz}$. The source level was approximately $183 \mathrm{~dB}$ re $1 \mu \mathrm{Pa}$ at $1 \mathrm{~m}$. The transmission is controlled manually from the command center on a ship. Typically, less than nine nodes were available at a given test. The reasons are: (1) Some of the nodes were outside the communication range of the mother ship, and (2) some of the nodes were moved by the fishermen and had to be retrieved and re-deployed again.

Each $m$-sequence contained 511 bits and lasted about $99.8 \mathrm{~ms}$. Ten $m$-sequences were transmitted in sequence from one modem with approximately $1.7 \mathrm{~s}$ separation between them, and then transmitted from the next modem by round robin. The received signals were matched filtered using the transmitted signal with different codes to identify its source. Knowing the source transmission time, one estimates the one-way transmission time. In a multipath environment, the impulse response determined from the matched filter will show many path arrivals. The travel time difference between reciprocal transmissions is determined from the travel time difference of the dominant (ray) path arrivals between reciprocal transmissions. While the one-way transmission time is dependent on which path one uses, the travel time difference is in principle the same no matter which path one uses, as long as the impulse responses remain approximate during the two-way transmission time. As a result, it is less sensitive to the path as long as the environment remains relatively unchanged. When many nodes were involved in one test, the round robin may take tens of seconds and the environment may have changed. In this case, the path arrival pattern may have changed between the reciprocal transmissions and the travel time difference measured by peak picking may be in error. For this test, such data are discarded. The lesson learned is to conduct the two-way transmissions (between a pair of nodes) one after the other immediately. The same remark applies to the node motions for nodes deployed in the water column. Consider, for example, distributed nodes separated at a nominal distance of, say, $3 \mathrm{~km}$. The two way transmissions can be completed in less than $6 \mathrm{~s}$, during which time the distance between the nodes can be assumed unchanged since node motion is generally slow; it usually take minutes or tens of minutes to complete a watch circle depending on the cable tension and rate of current change. One obtains one instantaneous measurement of the two-way travel time difference and node distance (from the mean travel time given the mean sound speed). Assuming the currents vary slowly with time, one can construct the current field using many such instantaneous measurements.

The purpose of this experiment is to study the feasibility of current mapping using the distributed sensors. Specifically, one attempts to (1) estimate the spatial 
distribution of the current within an area (for a limited time) and (2) estimate the temporal variation of the current for an extended time to compare with the ADCP data collected nearby. Due to experimental constraints, current distribution was only estimated for selected triangles of nodes. Consequently, Method 1 was applied to most of the data. The results are shown below.

\section{A. Measurement of the temporal variation of the current}

Figure 13(a) shows the in situ measurements of the surface current velocity from the weather buoy moored nearby the T4-T2 path as shown in Fig. 12. The data are reported as an average over the first 10-min time period of every hour. The acoustic travel time differences along the T4-T2 path were measured over many periods of time and computed to determine the mean current speed along this path. To make the comparison of two measurements, the current data from the weather buoy are projected onto the T4-T2 direction as shown in Fig. 13(b) to compare with the currents determined from the acoustic data. The two results are generally in good agreement - the estimated current shows a trend similar to the in situ measurements. Some discrepancies were observed, mostly during the changing tidal flow. It should be noted that acoustic measurements were the average current along the path whereas the in situ data were collected at a spot. Similarly, one estimated the current along the T4-T3 path using acoustic data. The results are shown in Fig. 13(c). Based on previous oceanographic measurements, the current in this area is dominated by the regular coastal tidal current
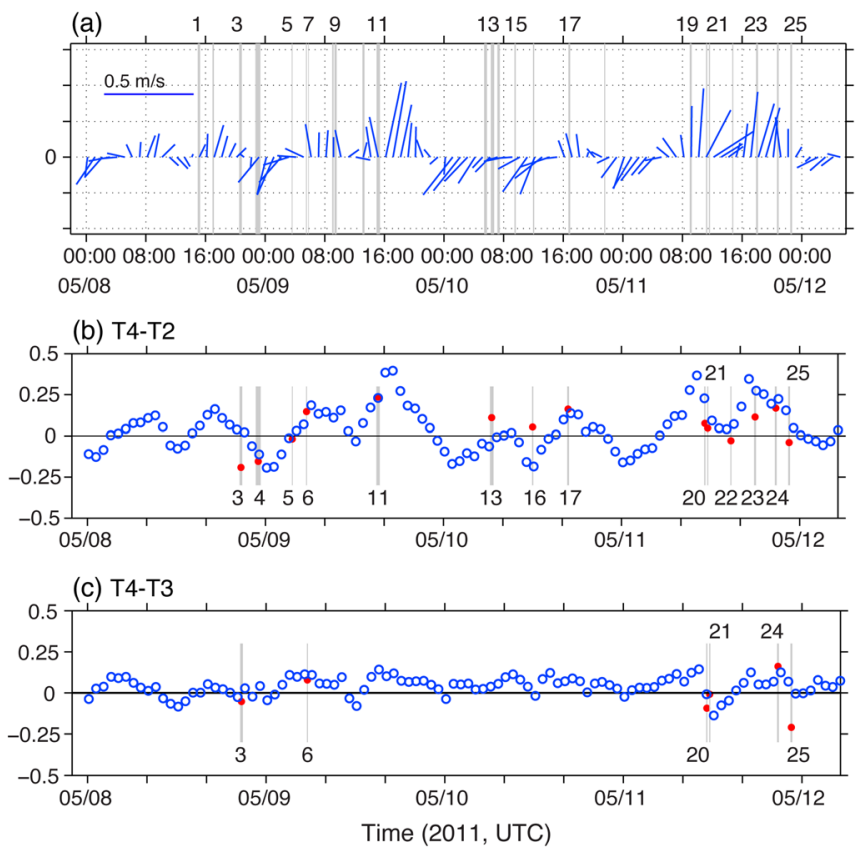

FIG. 13. (Color online) (a) The current vector measured by the weather buoy. The vertical line indicates the transmission time. (b) Temporal variation of mean current estimated along the T4-T2 path. (c) Temporal variation of mean current estimated along the T4-T3 path. Open and solid circles indicate the current measured by the weather buoy and by the reciprocal acoustic method, respectively. Positive value indicates the current moving away from T4. with the semi-diurnal period, flowing in a typical north-south direction along the isobaths. During the experiment, a typhoon named Aere approached Taiwan: According to the Weather Bureau, the storm center was located approximately $590 \mathrm{~km}$ south of Taiwan's southern tip on the morning of May 9 and moved in a northern direction and passed the east side of Taiwan (top panel of Fig. 12). Although the area was shielded by the Central Mountain Range, the current in the area might have been affected.

\section{B. Measurement of the spatial distribution of the current field}

The acoustic data from various tests (conducted at different times) were analyzed using the distributed sensing methods. The current field, linearly interpolated from the acoustic data, is shown by the arrows in Fig. 14 for different sensor locations. Figure 14 shows that the current flows along the isobaths from north-west and turns eastwards toward to the shore. These examples demonstrate that one can map out the spatial distribution of the current field. The mapping can be extended in principle to a bigger area using all the sensor nodes. To do so would require an acoustic network which can be commanded and controlled from a central node or an automatic command and control system based on time share. These systems are yet to be developed.

\section{SUMMARY AND CONCLUSIONS}

Conventional OAT systems spread the sensor nodes on the perimeter. By communicating signals between all sensors nodes, it creates measurements equivalent to that provided by an order $N^{2}$ virtual node. Distributed networked systems
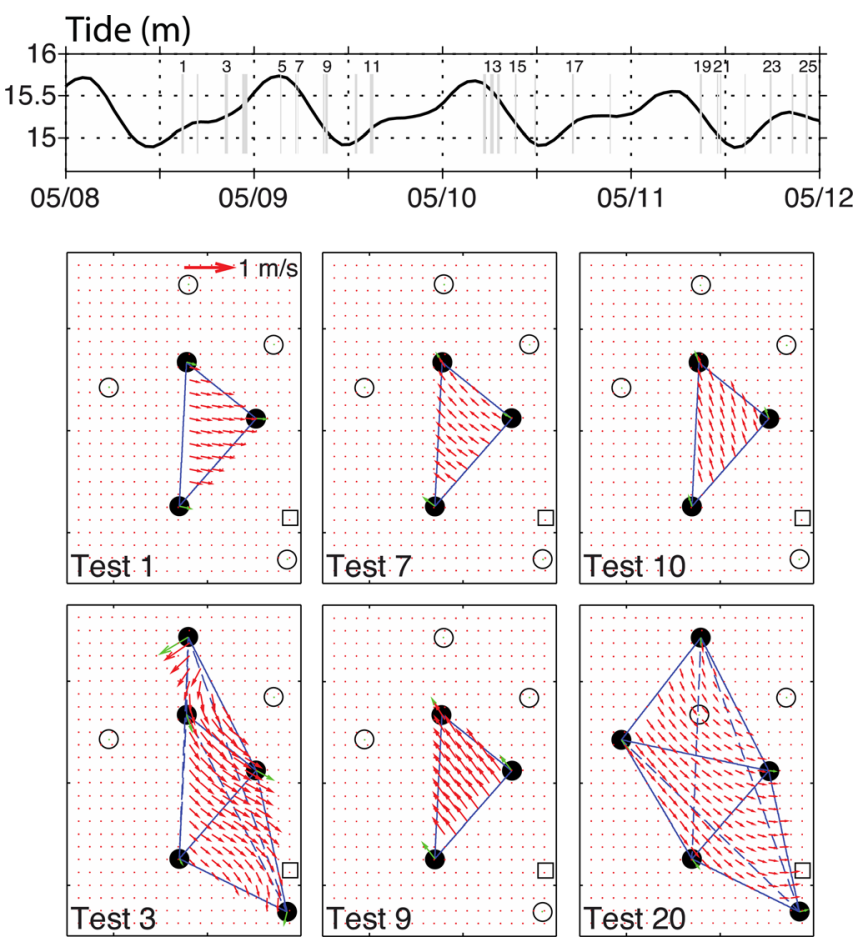

FIG. 14. (Color online) Inverted spatial distributions of the current field. The circles show the modem positions. The solid lines show the ray paths used for the inversion. 
use physical nodes to sample the field. Approximately how many distributed sensors are needed to provide the same quality of measurements is studied in this paper. The concept of distributed sensing becomes attractive when the cost of sensor nodes becomes small and insignificant in the system design (compared with other costs such as deployment and power cost). Distributed networked systems often use high frequency signals for communications due to the relatively short node distances between the nodes. Sensor configuration is automatically measured using the communication signals without requiring additional deployment of high frequency transponders as used in low frequency OAT systems. Significant savings in sensor deployment cost and deployment time can be expected due to the small sizes of the nodes. The transducer size decreases with increasing frequency and so does the hardware cost.

A major concern of a long term ocean measurement system is the power consumption which impacts the lifetime of the measurement system when powered by a battery. An attractive aspect of the distributed networked systems is that it uses much less projection power compared with conventional OAT systems at the same frequency due to the short node distances. Additional significant savings in power can be achieved for distributed systems by projecting signals at higher frequencies. Power analysis for signal projection shows a potential savings by as much as $15 \mathrm{~dB}$, which is very significant. Note that higher frequencies provide larger BWs and hence better time resolution.

Performances (fractional residual error variance) of distributed systems are evaluated using tomographic inversion methods to compare with that obtained with the conventional OAT systems. For tomographic inversion, one generally faces an underdetermined system. Better performance can be achieved given certain prior knowledge of the current field (in terms of wavenumber spectrum spread and shape) to design an optimal regulator. Conversely, incorrect prior knowledge can lead to a model mismatch problem. This applies to distributed systems as well as the conventional OAT system. While selecting an optimal regulator is important for tomographic inversion, it is shown that for distributed systems a substantial performance improvement can be gained by increasing the number of sensors, which more than compensates for the loss due to lack of prior knowledge about the data. For example, using 16 nodes one obtains a factor of $\sim 48$ performance improvement (with a simple regulator), compared with the best result using the 8-nodes OAT system with an optimal regulator tailored for a narrowband case.

Rapid in-buoy processing algorithms are proposed for distributed systems assuming that the nodes distances are sufficiently small (i.e., smaller than the correlation length of the current field). This algorithm does not employ a regulator and thus does not take advantage of any prior knowledge of the current field. For distributed systems, sensor nodes are divided into triangular clusters. Method 1 assumes that the differences of currents at different locations within the triangle (spatial variation of the current) are much smaller than the current itself, which is a good assumption when the current gradient is small within the triangle. When current gradient is non-negligible but is a constant within a cluster of triangles, one would use only the current estimated at the (inner) nodes obtained by averaging the currents from the neighboring sub-triangles and not use the latter (sub-triangles) to construct the final field. This method, Method 2, which keeps part of the data obtained in Method 1, is shown to correct for most of the errors incurred in Method 1 for the narrowband current case. It yields similar results as Method 1 for the broadband case. Data processing is simple and can be performed in individual nodes, via e.g., in-buoy processing, without requiring a model representation of the current field, or additional constraints such as the Tikhonov regularization. The fractional error variance so obtained is similar to that obtained by tomographic inversion assuming a simple regulator (no prior knowledge). Much improved fractional error variance can be obtained by adding more sensors.

Experimental data collected at sea were analyzed using distributed signal processing to demonstrate the feasibility of using distributed sensors for current mapping. For this experiment, due to hardware and software constraints, the demonstration was done over a small area involving a small number of nodes and for a limited time window. Nonetheless, it demonstrates the capability of the method for extended area and time.

Distributed sensing offers other advantages such as adaptive topology and mapping resolution, easy deployment and position survey, etc., as discussed in Sec. II above, which could be important from the system point of view.

\section{ACKNOWLEDGMENT}

This work is supported by the Office of Naval Research, USA, and National Science Council of Taiwan, R.O.C. through Contract Nos. 101-2611-M-002-007 and 101-2218-E-110-001. The authors would like to thank the reviewers for many useful comments that helped to improve the manuscript. We are furthermore indebted to Professor Ling-Yun Chiao, National Taiwan University for fruitful discussions.

${ }^{1}$ T. O. T. Group, D. Behringer, T. Birdsall, M. Brown, B. Cornuelle, R. Heinmiller, R. Knox, K. Metzger, W. Munk, J. Spiesberger, R. Spindel, D. Webb, P. Worcester, and C. Wunsch, "A demonstration of ocean acoustic tomography," Nature 299, 121-125 (1982).

${ }^{2}$ W. Munk, P. Worcester, and C. Wunsch, Ocean Acoustic Tomography (Cambridge University Press, Cambridge, 1995), Chap. 3, pp. 116-135 and 233-234.

${ }^{3}$ B. Cornuelle, W. Munk, and P. Worcester, "Ocean acoustic tomography from ships,” J. Geophys. Res. 94, 6232-6250, doi:10.1029/JC094iC05p06232 (1989).

${ }^{4}$ J.-H. Park and A. Kaneko, "Assimilation of coastal acoustic tomography data into a barotropic ocean model," Geophys. Res. Lett. 27, 3373-3376, doi:10.1029/2000GL011600 (2000).

${ }^{5}$ P. Elisseeff, H. Schmidt, M. Johnson, D. Herold, N. R. Chapman, and M. M. McDonald, "Acoustic tomography of a coastal front in Haro Strait, British Columbia,” J. Acoust. Soc. Am. 106, 169-184 (1999).

${ }^{6}$ K. Yamaguchi, J. Lin, A. Kaneko, T. Yayamoto, N. Gohda, H.-Q. Nguyen, and H. Zheng, "A continuous mapping of tidal current structures in the Kanmon Strait,” J. Oceanogr. 61, 283-294 (2005).

${ }^{7}$ X.-H. Zhu, A. Kaneko, Q. Wu, C. Zhang, N. Taniguchi, and N. Gohda, "Mapping tidal current structures in Zhitouyang Bay, China, using coastal acoustic tomography,” IEEE J. Ocean. Eng. 38, 285-296 (2013).

${ }^{8}$ F. Akyildiz, D. Pompili, and T. Melodia, "Underwater acoustic sensor networks: Research challenges," in Ad Hoc Networks (Elsevier, New York, 2005), Vol. 3, pp. 257-279. 
${ }^{9}$ M. Stojanovic, "Capacity of a relay acoustic channel," in OCEANS 2007 (2007), pp. 1-7.

${ }^{10}$ P.-O. Persson and G. Strang, "A simple mesh generator in MATLAB," SIAM Rev. 46, 329-345 (2004).

${ }^{11} \mathrm{P}$. M. Shearer, Introduction to Seismology (Cambridge University Press, Cambridge, 2009), Chap. 5.

${ }^{12}$ M. A. Ainslie and J. G. McColm, "A simplified formula for viscous and chemical absorption in sea water,” J. Acoust. Soc. Am. 103, 1671-1672 (1998).

${ }^{13} \mathrm{~F}$. Gaillard, "Evaluating the information content of tomographic data: Application to mesoscale observations," J. Geophys. Res. 97, 15489-15505, doi:10.1029/92JC01295 (1992).
${ }^{14}$ P. C. Hansen and D. P. O'Leary, "The use of the L-curve in the regularization of discrete ill-posed problems," SIAM J. Sci. Comput. (USA) 14, 1487-1503 (1993).

${ }^{15}$ L.-Y. Chiao and B.-Y. Kuo, "Multiscale seismic tomography," Geophys. J. Int. 145, 517-527 (2001).

${ }^{16}$ J. Trampert and R. Snieder, "Model estimations biased by truncated expansions: Possible artifacts in seismic tomography," Science 271, 1257-1260 (1996).

${ }^{17}$ J. K. Lewis, J. Rudzinsky, S. Rajan, P. J. Stein, A. Vandiver, and K. Group, "Model-oriented ocean tomography using higher frequency, bottommounted hydrophones," J. Acoust. Soc. Am. 117, 3539-3554 (2005). 